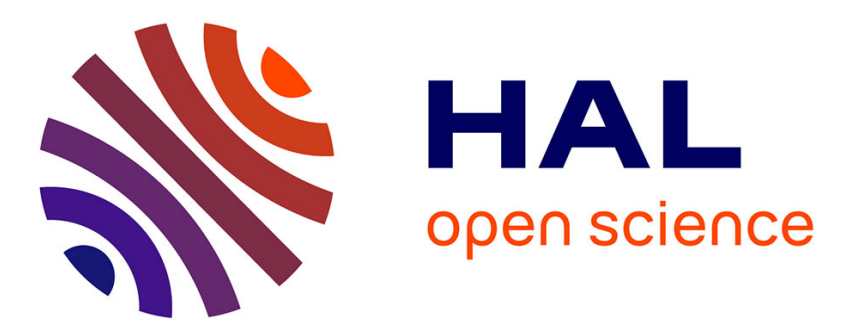

\title{
Application of scattering theories to the characterization of precipitation processes
}

Sandra Jacquier, Frédéric Gruy

\section{To cite this version:}

Sandra Jacquier, Frédéric Gruy. Application of scattering theories to the characterization of precipitation processes. Light Scattering Reviews 5, Springer, pp.37-78, 2010, Single Light Scattering and Radiative Transfer, 978-3-642-10335-3. 10.1007/978-3-642-10336-0 . emse-03419758

\section{HAL Id: emse-03419758 \\ https://hal-emse.ccsd.cnrs.fr/emse-03419758}

Submitted on 8 Nov 2021

HAL is a multi-disciplinary open access archive for the deposit and dissemination of scientific research documents, whether they are published or not. The documents may come from teaching and research institutions in France or abroad, or from public or private research centers.
L'archive ouverte pluridisciplinaire $\mathbf{H A L}$, est destinée au dépôt et à la diffusion de documents scientifiques de niveau recherche, publiés ou non, émanant des établissements d'enseignement et de recherche français ou étrangers, des laboratoires publics ou privés. 


\title{
Application of scattering theories to the characterization of precipitation processes
}

\author{
Sandra Jacquier and Frédéric Gruy \\ Ecole Nationale Supérieure des Mines, \\ 158 Cours Fauriel, 42023 Saint-Etienne \\ jacquier@emse.fr, gruy@emse.fr
}

\section{Introduction}

The solid-liquid suspensions are frequently used in industrial processes. These suspensions usually contain aggregates made up of solid primary particles. Many characterization tools of these suspensions are based on light scattering (Mie theory). However, the Mie theory (1908) is not always applicable to the practical problems since the scatterer must be a homogeneous sphere. The ordinary particle sizers that use this theory do not make it possible to measure the non-spherical particle geometrical characteristics. Extensions of the Mie theory for arbitrary shaped particles or particle aggregates are available nowadays (T-Matrix method, Generalized Multiparticle Mie (GMM)-solution, etc.). But the computing times of the optical properties via these exact theories do not allow for a real-time analysis. This chapter is thus dedicated towards the search of approximate methods for the estimate of aggregate optical properties, particularly their scattering cross section.

This chapter is split into five parts. The first one (section 2) concerns the aggregation process and, more generally, the precipitation process, to provide a better understanding of the framework of this study. Precipitation is the formation of a solid in a solution during a chemical reaction. Morphology of particles currently observed during precipitation or particle 
synthesis will be described. Relationship between optics and particle technology will be recalled. The third section outlines briefly the different approximate methods used for the case of spherical and non-spherical particles. The fourth section presents selected approximations for light scattering cross sections in the case of aggregates. The quality of each approximation will be discussed by comparison with the exact theory. Practical cases will be presented. The fifth section is an extension of the previous section to the light pressure cross section. The last section is an attempt to relate the scattering properties of aggregates to their geometrical characteristics.

In the next section we describe the context of the need of approximate methods for scattering cross section of aggregates.

\section{Aggregate formation}

Materials are often made from inorganic particles. These are formed by reactions in gas phase or more commonly in liquid phases. The main process is named precipitation.

\subsection{Precipitation and particles synthesis}

The classical situation is the following: a solution consisting of a solute A and a solvent is mixed with another one consisting of a solute B and the same solvent. The two solutes react to form a solid product denoted AB.

$\mathrm{A}+\mathrm{B} \leftrightarrow \mathrm{AB}$

$\mathrm{A}$ and $\mathrm{B}$ are often ions.

If the equilibrium between the solid phase and the solutes is reached, thermodynamics tells us that this equilibrium (saturated solution) is characterized by the solubility of the solid phase.

However, when the concentrations of A and B are high enough to produce solid particles, the initial solution is supersaturated. So, the ratio of the actual concentration and the equilibrium concentration (solubility), called supersaturation, is the key parameter of the dynamics of precipitation. Higher is the supersaturation, higher is the precipitation rate. Particle formation can be distinguished into three stages: nucleation, growth and agglomeration. 
Nucleation is the birth of particles that are large enough to grow (nuclei). Too small particles are unstable and dissolve in the solution. Nucleation rate $\mathrm{J}_{\mathrm{N}}$ is defined as the number of created nuclei per unit volume and per unit time. The formed nuclei are characterized by a socalled critical size. Typical critical size values are within the range [1 $\mathrm{nm}-50 \mathrm{~nm}]$. The nucleation rate is a non linear increasing function of supersaturation. Nucleation stage in a precipitation process is often difficult to observe. If light is used as a probe to study precipitation, nuclei can be treated as Rayleigh scatterers. Considering the nuclei concentration, interaction between light and suspension is usually below the detector threshold. Other methods (for instance, Small-Angle X ray Scattering) are preferred, but are more difficult to be applied. Details about the nuclei are not known. Hence, nuclei leading to inorganic particles are often considered as amorphous and spherical.

The following stage is the growth of the nuclei. Additionally, they may convert to crystals. Crystals present facets, the occurrence of which can be explained from crystallography and thermodynamics. However, depending on the precipitation reaction, only amorphous particles (i.e., hydroxylated compounds) can be found. Usually, the growth rate is defined as the derivative of crystal characteristic length (e.g, the diameter) with time. The growth rate increases with the supersaturation. The most often, the relation between growth rate and supersaturation is linear.

For different reasons (one is the decrease of supersaturation during precipitation, due to the mass transfer from solution to crystals), an upper size limit for the growing crystals is observed. At the end of the second stage, crystal or particle size ranges between $0.1 \mu \mathrm{m}$ and $10 \mu \mathrm{m}$. During this period, particles become large enough to scatter light effectively. Thus, light scattering methods are suitable for studying the growth of crystals or primary particles in suspension.

Before or after reaching the end of growth, crystals or amorphous particles can form clusters or agglomerates. Agglomeration requires the collision of particles and their subsequent 
adhesion due to attractive forces (for instance, Van der Waals forces). Consolidation between primary particles or crystals can take place by crystalline growth from the contact point.

In quiescent liquids, the particle collisions are due to their Brownian motion. For flowing suspensions, collisions are due to Brownian motion for small particles or crystals (smaller than about $0.2 \mu \mathrm{m}$ ) and due to local shear for larger particles.

When the agglomerate increases in size, it becomes fragile. Then, break up takes place and a limit size is reached (with values in the range [5 $\mu \mathrm{m}-100 \mu \mathrm{m}]$ ). The stress acting on the agglomerate results from the shear, but also the transition between viscous and inertial turbulent regimes. This leads to agglomerates containing often a few primary particles (less than one hundred primary particles).

Summarizing: inorganic solutes can lead to solid particles in a batch precipitation vessel, provided that the solution is initially supersaturated. Nucleation, i.e. the birth of nuclei with a critical size, takes place, while supersaturation decreases as a consequence. Finally, supersaturation becomes too small, to produce new nuclei. Thus, there is a mass transfer from solution to the nuclei surface leading to the growth of nuclei. In this way, crystals or amorphous particles are formed. As supersaturation tends to one, growth stops. Depending on the surface charge of particle, their agglomeration may occur for the growth period.

\subsection{Particles shapes during precipitation}

Images from electron microscopy often show the complexity of the particles structure. Particles formed by growth from solution can be crystals with well defined crystalline faces, but may be agglomerates of smaller (nanometric) particles. In this case, agglomeration can be due to Brownian motion and the subsequent collision of particles, but also the contact of specific crystalline faces belonging to two particles. The first situation leads to random agglomerate with spherical symmetry. The second phenomenon, also called orientated agglomeration, leads to regularly shaped particles (i.e., cylinder as disks stacking). It must be underlined that the mechanism of orientated agglomeration is still being studied. Whatever the structure of particles: crystals, random or ordered agglomerate of nanoparticles, their geometric characteristics are easily determined by image analysis of electron micrographs. 
However, these particles are rarely apart. They form agglomerates after collision due to Brownian motion and / or shear flow. The structure of agglomerates is disordered and is often considered as fractal-like. However, the reader must keep in mind that these agglomerates consist of only a few particles.

Figure 1 presents agglomerates of $\mathrm{SrMoO}_{4}$ strontium molybdate crystals (Cameirao et al, 2008). They are obtained by precipitation:

$\mathrm{SrCl}_{2}+\mathrm{Na}_{2} \mathrm{MoO}_{4} \rightarrow \mathrm{SrMoO}_{4}+2 \mathrm{NaCl}$

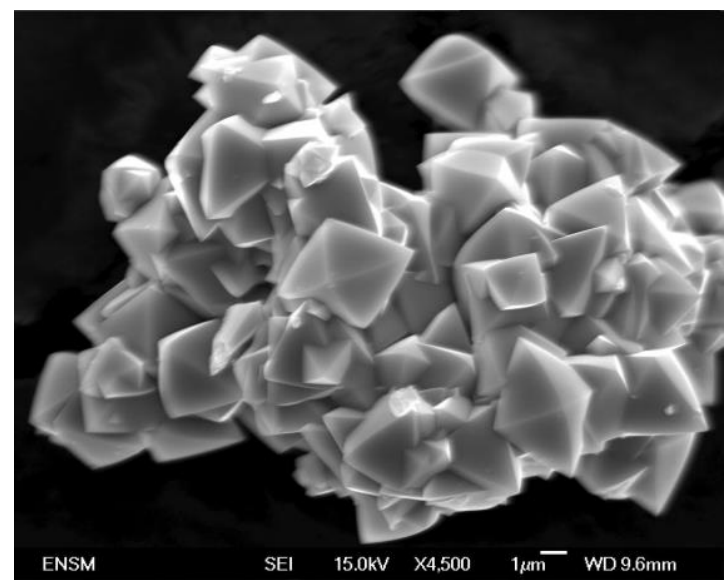

Fig. 1. Agglomerate of $\mathrm{SrMoO}_{4}$ crystals

Bipyramidal crystals in the size range [3-10 $\mu \mathrm{m}]$ are formed. Agglomerates size is in the range $[20-80 \mu \mathrm{m}]$.

Figure 2 presents agglomerates of zinc sulphide particles (Mekki-Berrada et al, 2005). They are obtained by homogeneous precipitation:

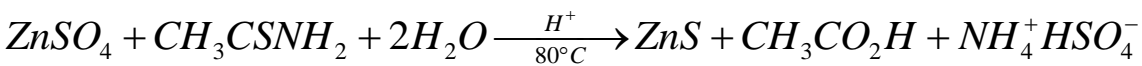




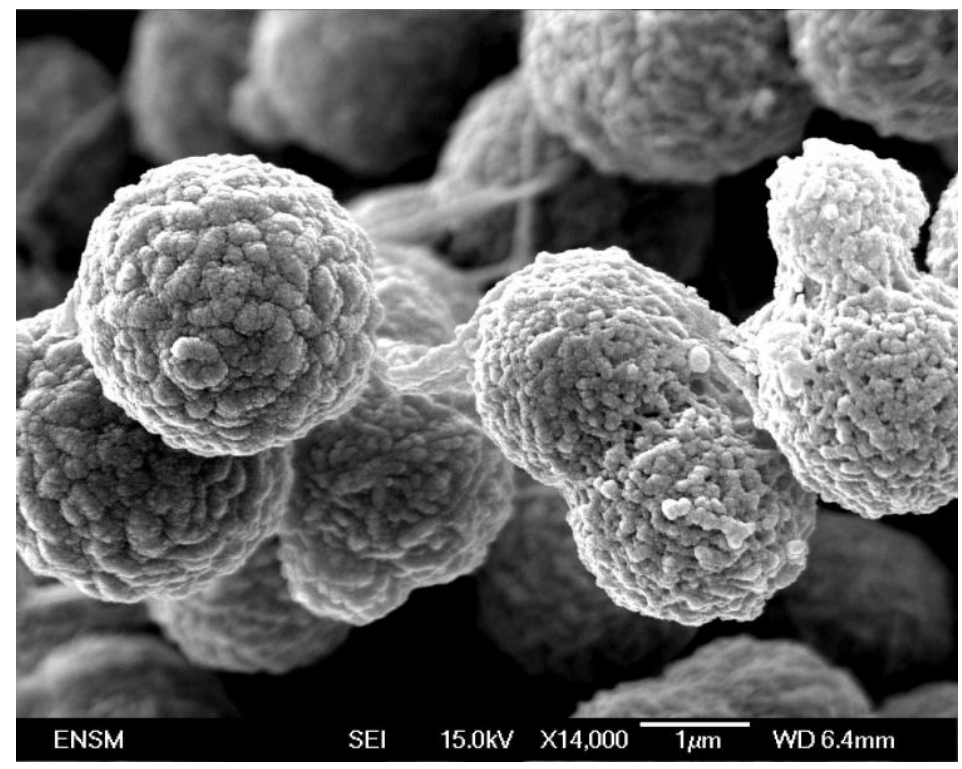

Fig. 2. Multi-scale ZnS agglomerate

$\mathrm{ZnS}$ particles are sphere-like with a mean size equal to $3 \mu \mathrm{m}$. They consist of nanoparticles, $30 \mathrm{~nm}$ sized (one may observe an intermediate structure in the range $100 . .300 \mathrm{~nm}$ ). Microparticles seem relatively dense. However, porosity and inner structure depend on the acidity and temperature. Micro-particles collide to form agglomerates in the range [20 - $60 \mu \mathrm{m}]$.

Figure 3 shows ordered agglomerates resulting from stacking of $\mathrm{Ni}(\mathrm{OH})_{2}$ nanosized disks (Coudun et al., 2007). They are obtained by precipitation from nickel di-dodecylsulfate and ammonia:

$\mathrm{Ni}(\mathrm{DS})_{2}+2 \mathrm{OH} \mathrm{H}^{-} \stackrel{\mathrm{NH}_{3}}{60^{\circ} \mathrm{C}} \mathrm{Ni}(\mathrm{OH})_{2}+2 \mathrm{DS}^{-}$

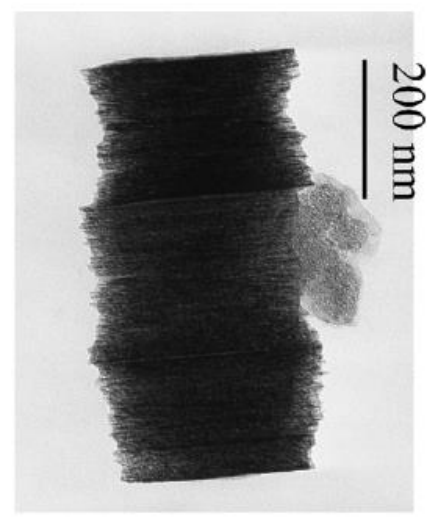

Fig. 3. $\mathrm{Ni}(\mathrm{OH})_{2}$ nanosized agglomerates 


\subsection{Dynamics of precipitation: modelling}

In order to manage the complexity of precipitation dynamics, each particle in the precipitation reactor is characterized by space coordinates $(x, y, z)$ and internal coordinates $p_{i}$ (i.e. radius, characteristic lengths of crystal, volume, porosity, gyration radius, fractal dimension, ...) with $1 \leq i \leq P$.

The largest being is the number $P$, more comprehensive is the description of particles. So, the population density function $\mathrm{n}\left(\mathrm{x}, \mathrm{y}, \mathrm{z}, \mathrm{p}_{1} \ldots \mathrm{p}_{\mathrm{P}}\right)$ is such as $\mathrm{dN}=\mathrm{n} d \mathrm{dx} d \mathrm{dz} \mathrm{dp}_{1} \ldots d \mathrm{p}_{\mathrm{P}}$ represents the number of particles with $x$ in the range $[x ; x+d x], p_{i}$ in the range $\left[p_{i} p_{i}+d p_{i}\right]$.

This population density obeys the population balance equation (PBE), that can be formally written as:

$\frac{\partial n}{\partial t}=-\vec{\nabla} \cdot(\vec{v} n)-\sum_{i=1}^{P} \frac{\partial}{\partial p_{i}}\left(G_{i}(S) n\right)+J_{N}(S) \delta\left(p_{1}-p_{1 c}\right) \ldots \delta\left(p_{P}-p_{P_{c}}\right)+V_{a g}$

where

$\vec{v}$ is the particle velocity,

$t$ is the time,

$G_{i}$ is the growth rate for the $\mathrm{p}_{\mathrm{i}}$ parameter $\left(G_{i}=\frac{d p_{i}}{d t}\right)$

$\delta$ is the Dirac function, $p_{i c}$ is the internal parameter corresponding to the critical nucleus,

$J_{N}(S)$ is the nucleation rate as a function of the supersaturation $S$,

$V_{a g}$ is the agglomeration rate.

More often, the following assumptions are used:

- homogeneous suspension

- only one internal parameter (particle radius) 
- agglomeration taken into account only at the end of nucleation and growth

- fractal-like agglomerates with constant fractal dimension.

For fractal-like agglomerates, the relation between the number $j$ of primary particles inside the agglomerate and its outer radius $R_{j}$ is:

$$
R_{j}=a\left(\frac{j}{S_{r}}\right)^{1 / D_{F}}
$$

$a$ is the radius of the primary particle. $D_{F}$ and $S_{r}$ are respectively the fractal dimension and the structure factor, which is a function of $D_{F}$.

Attempts to consider two internal parameters (radius and length for particles as a needle, solid volume and surface area for porous particles) have also been made (Tandon and Rosner, 1999; Kostoglou et al., 2006).

Summing up, precipitated particles have a multi-scale structure. One commonly observes three characteristic length values: $20 \mathrm{~nm}, 2 \mu \mathrm{m}, 20 \mu \mathrm{m}$. Sometimes, only two $(2 \mu \mathrm{m}, 20 \mu \mathrm{m})$ are observed. The smallest particles are most often dense and spherical. The intermediate particles are relatively dense and have a well-defined shape (i.e. sphere, cylinder, ellipsoid, etc). The largest scale corresponds to disordered or random agglomerates. The reader interested in details of precipitation and population balance may refer to Sugimoto (2000) and Randolph and Larson (1988). 


\subsection{Particle sizing during precipitation}

Considerable efforts are made to understand the precipitation mechanisms and to predict the change of the particle morphology with time. On the other hand, industrialists need to monitor and control the precipitation process. Off line size measurements (i.e. using filtration, powder drying and Scanning Electron Microscope (SEM) observations or suspension sampling and sizing with granulometers) are now avoided because these operations modify the particle morphology. On-line measurements (i.e., using a recirculation loop with a measurement cell in a granulometer) are possible, but representative sampling is difficult to carry out. In line measurements are preferred. They are often based on light extinction and are obtained from turbidimetric sensor. So, turbidimetry will be at the centre of this chapter.

Typically, the optical sensor for particle sizing is not the only one in the precipitating suspension. The temperature probe and concentration sensors are always introduced within. Thus, supersaturation and solid fraction (via mass balancing from solute concentration) are deduced and, as a consequence, are known.

A turbidimetric sensor records the transmitted light intensity $\mathrm{I}_{\mathrm{t}}$. One defines the extinction coefficient or turbidity ${ }^{1}$ as (see, for instance, Elimelech et al., 1995):

$\tau=-\frac{1}{L} \ln \frac{I_{t}}{I_{0}}$

$I_{o}$ and $L$ are the incident light intensity and the geometrical thickness of the medium, respectively. For a monodisperse diluted suspension, the turbidity obeys the equation:

$$
\tau(\lambda)=N\left(x_{s}, y_{s}, z_{s}, p_{1} \ldots p_{P}\right) C_{e x t}\left(\lambda, p_{1} \ldots p_{P}\right)
$$

\footnotetext{
${ }^{1}$ Definitions and notations in Eqs. 3-5 are used by researchers in the field of particle sizing concerning suspensions. Other researchers prefer these ones: $\tau^{*}=-\ln \left(I_{t} / I_{0}\right)$ and $\sigma_{e x t}(\lambda)=\tau^{*}(\lambda) / L$ where $\tau^{*}$ and $\sigma_{e x t}$ are the optical thickness and the extinction coefficient, respectively.
} 
The functions $N$ and $C_{\text {ext }}$ are the particle number concentration and the extinction cross section of the particles. $\left(x_{s}, y_{s}, z_{s}\right)$ are the coordinates of the sensor in the reactor.

For a complex diluted suspension, turbidity contains the contribution of each kind of particles:

$$
\tau(\lambda)=\int_{\left[p_{1} \ldots p_{P}\right]} n\left(x_{s}, y_{s}, z_{s}, p_{1} \ldots p_{P}\right) C_{e x t}\left(\lambda_{i} p_{1} \ldots p_{P}\right) d p_{1} \ldots d p_{P}
$$

Thus, the turbidity monitoring gives information on the population density change with time during the precipitation process.

Two strategies can be considered for the analysis of turbidity signals:

- inversion of the integral equation (Eq.5) in order to get the population density. Then, comparison to PBE (Eq.1) solution and identification of unknown physico-chemical and morphological parameters (i.e., fractal dimension ...)

- PBE (Eq.1) solving; calculation of $\tau(\lambda)$ (Eq.5); comparison with experimental turbidity for deducing the unknown physico-chemical and morphological parameters.

For numerical reasons, the last one is preferred. However, whatever the strategy, the knowledge of $\mathrm{C}_{\mathrm{ext}}\left(\lambda, \mathrm{p}_{1} \ldots \mathrm{p}_{\mathrm{P}}\right)$ is needed.

The extinction cross section is dependent on the relative refractive index $m$, which is the ratio between refractive indices of material and suspending medium. Three typical cases can be envisaged: low optical contrast $m-1$ value $(0<m-1<0.1)$, moderate contrast $m-1$ value 
$(0.1<m-1<0.5)$ and high optical constrast $m-1$ value $(m-1>0.5)$. Corresponding materials (suspended in water) could be, respectively, silica $\mathrm{SiO}_{2}(m=1.08)$, alumina $(m=$ 1.20) and titania $\mathrm{TiO}_{2}(m=2)$.

We will focus our work on non-absorbing (in the visible range) materials that are most commonly found in the precipitation process. Thus, scattering $\mathrm{C}_{\mathrm{sca}}$ and extinction $\mathrm{C}_{\mathrm{ext}}$ cross sections are equal.

It is obvious that PBE solving, cross sections calculation and optimization procedure require great computational efforts and make it difficult to get results in a short time. Therefore, any rapid calculation of the optical cross sections would be a progress. The accuracy of approximations has to fit the measurement accuracy. In the case of turbidity, measurements within $3 \%$ error can be considered as satisfactory.

The need for approximations is particularly important for agglomerates ${ }^{2}$. Fast calculations have to be based on known approximations coming from the light scattering theory. Next chapter shortly recalls them.

\section{Approximations for non-spherical particles}

The scattering cross section is a function of the dimensionless particle size parameter $x(=k a$ for a sphere), the particle and the medium optical refractive indices respectively denoted $n_{p}$ and $n_{m}$, the wavelength $\lambda$ (and the wave number $k=2 \pi / \lambda$ ) of the incident light in the medium and the orientation of the incident light in the relation of the particle position. The

\footnotetext{
2 The name aggregation corresponds to the formation of a cluster, the primary particles of which only interact by physical forces as Van der Waals forces. On the other hand, agglomeration is aggregation followed by strengthening at the contact point in a supersaturated solution. Aggregate and agglomerate optical properties will be treated in the same way.
} 
relative refractive index $m$, which is used in the following equations, is the ratio between the material refractive index $n_{p}$ and the medium refractive index $n_{m}$.

The exact theory was developed for a sphere in 1908 by G. Mie (cf. (Hulst 1981)) and for spheroids by several authors (Asano and Yamamoto, 1975, Asano, 1979, Asano and Sato, 1980, Voshchinnikov and Farafonov, 1992, Farafonov et al., 1999).

In this section, three classical approximations are recalled: the Rayleigh approximation, the Rayleigh-Gans-Debye approximation and the Anomalous Diffraction approximation. Principles are presented and application is given for a sphere. The reader interested in by details on scattering theories may refer to Van de Hulst (1981) and Kokhanovsky (2004).

\subsection{Rayleigh approximation}

The Rayleigh approximation that considers the scatterer as an oscillating dipole has a validity range of $x<<1,|m x| \square 1$.

So, the scattering efficiency factor for a sphere is:

$Q_{s c a}=\frac{8}{3} x^{4}\left|\frac{m^{2}-1}{m^{2}+2}\right|^{2}$

and the scattering cross section is $C_{s c a}=Q_{s c a} G$ ( $G$ represents the particle projected area, for a sphere $\left.G=\pi a^{2}\right)$.

A comparison between this approximation and the Mie exact theory shows that the validity range, in terms of maximum size, varies according to the relative refractive index and the scattering angle (Mishchenko et al., 2002, 2004).

\subsection{Rayleigh-Gans-Debye approximation}

The validity range of the Rayleigh-Gans-Debye approximation (RGD) is: $2 x|m-1|<<1$ and $|m-1|<<1$.

Figure 4 represents a particle with an unspecified shape lit by a plane wave being propagated along the axis z'. It is supposed that each volume element is a Rayleigh scatterer and behaves independently of the other particle volume elements. The scattering waves of all these volume 
elements interfere. The phases of all these scattering waves are ascribed in a common coordinates reference in order to handle their amplitude.
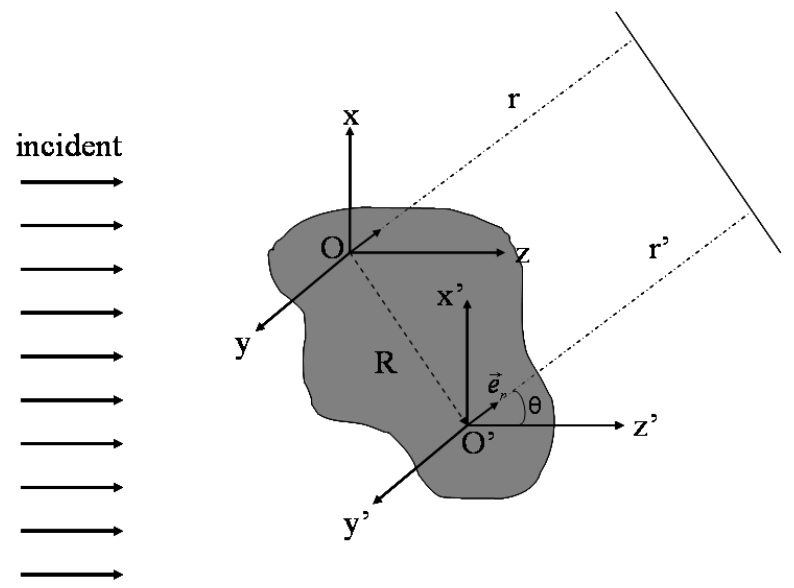

Fig. 4. RGD approximation

The expression of the contribution, of the volume element $\Delta V$ located out of $\mathrm{O}$, to the scattering field by the particle is:

$$
\left(\begin{array}{l}
\Delta E_{\mathrm{ll}, s c a} \\
\Delta E_{\perp, s c a}
\end{array}\right)=\left(\begin{array}{cc}
S_{2} & 0 \\
0 & S_{1}
\end{array}\right) \cdot \frac{e^{\mathrm{i} \cdot k \cdot r-\mathrm{i} \cdot k \cdot z}}{-\mathrm{i} \cdot k \cdot r} \cdot \Delta V\left(\begin{array}{l}
E_{\mathrm{ll}, i n c} \\
E_{\perp, i n c}
\end{array}\right)
$$

The contribution of a volume element located in $\mathrm{O}^{\prime}$ will be:

$\left(\begin{array}{l}\Delta E_{\mathrm{ll}, s c a} \\ \Delta E_{\perp, s c a}\end{array}\right)=\left(\begin{array}{cc}S_{2} & 0 \\ 0 & S_{1}\end{array}\right) \cdot \frac{e^{\mathrm{i} \cdot k(r-z)}}{-\mathrm{i} \cdot k \cdot r} \cdot \Delta V e^{i \delta}\left(\begin{array}{l}E_{\mathrm{ll}, i n c} \\ E_{\perp, i n c}\end{array}\right)$

with $\delta=k \vec{R}\left(\vec{e}_{z}-\vec{e}_{r}\right)$ and $\vec{R}=\overrightarrow{O O^{\prime}} . S_{1}$ and $S_{2}$ are the amplitude functions per volume unity:

$S_{1} \approx-\frac{i k^{3}}{2 \pi}(m-1)$ and $S_{2} \approx-\frac{i k^{3}}{2 \pi}(m-1) \cos \theta$.

$\theta$ is the scattering angle.

Integration is done with respect to particle volume $V$ to obtain the total field in the direction $\vec{e}_{r}$. So, the amplitude functions for the particle are: 
$S_{1}=-\frac{i k^{3}}{2 \pi}(m-1) V f$ and $S_{2}=-\frac{i k^{3}}{2 \pi}(m-1) V f \cos \theta$

The form factor $f$ obeys:

$f=\frac{1}{V} \int_{V} e^{i \delta} d V$

that becomes for a sphere:

$f(u)=\frac{3}{u^{3}}(\sin u-u \cos u), u=2 x \sin \frac{\theta}{2}$.

It follows for a spherical particle (Van de Hulst, 1981):

$Q_{s c a}=\int_{0}^{\pi} F(\theta) d \theta /\left(\pi a^{2}\right)$

where,

$F(\theta)=\pi a^{2} \frac{4}{9}|m-1|^{2} x^{4} f^{2}\left(2 x \sin \frac{\theta}{2}\right)\left(1+\cos ^{2} \theta\right) \sin \theta$

\subsection{Anomalous Diffraction approximation}

This approximation, due to Van de Hulst, bears the name of anomalous diffraction (AD) because for low optical contrast, the light passing through the particle (transmitted without deflection) interferes with that diffracted, then producing a diffraction known as anomalous. Let us consider particles such as: $x>>1$ and $\left|m_{r}-1\right|<<1$ (see the discussion of Videen and Chylek (1998) and Liu (1998)).

The second condition implies that the rays are not deviated when they cross the interface particle-medium and that the reflection is negligible with the same interface. Extinction is thus due to:

- absorption of the light passing through the particles

- interferences between the light passing through the particle and that passing around. 


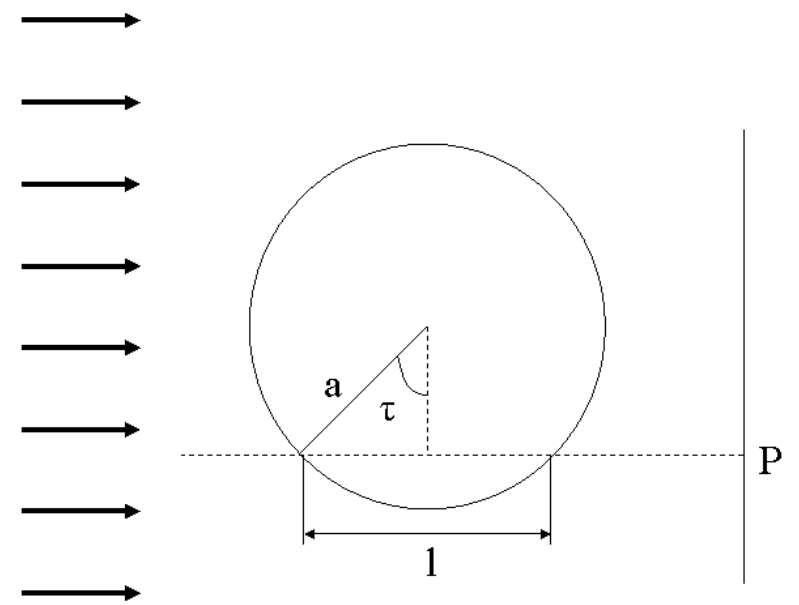

Fig. 5. Anomalous Diffraction approximation; ray passing through a sphere

Following Van de Hulst (1981), one derives:

$C_{\text {ext }}=\frac{4 \pi}{k^{2}} \mathfrak{R} e\{S(0)\}$ with $S(0)=\frac{k^{2}}{2 \pi} \iint_{\left[S_{p}\right]}\left(1-e^{-i k(m-1) l}\right) d y d z$

Integration is performed over the particle projected area $S_{p}$ on a plane perpendicular to propagation direction. $l$ is the computed path of light through the particle, which is a function of the projection coordinates $x$ and $y$.

The integrand represents the subtraction with "the part of shade" (value 1) of the rays passing through the particle $\left(e^{-i k(m-1) l}\right)$.

If $m$ is real,

$C_{s c a}=2 \iint_{\left[S_{p}\right]}[1-\cos (k l(m-1))] d S_{p}$.

Thus, it follows for a sphere

$Q_{s c a}=Q_{e x t}=2-\frac{4}{\rho} \sin \rho+\frac{4}{\rho^{2}}(1-\cos \rho)$

where $\rho=2 x(m-1)$.

The anomalous diffraction was applied to a sphere and an infinitely long circular cylinder (Van de Hulst, 1981), a prism column (Chylek and Klett, 1991), a hexagonal crystal of ice 
(Sun and Fu, 1999), ellipsoids (Lopatin and Sid'ko, 1988; Streekstra et al., 1994), a short cylinder (Liu et al,. 1998) and other various forms (Sun and Fu, 2001, Yang et al., 2004).

A comparison between AD and the exact theory (Liu et al., 1998) suggests that AD estimates the extinction more precisely in the case of a random orientation of nonspherical particles than for spheres.

The next section treats approximations for the case of the clusters of spheres. The derived approximations are directly related to the previous ones.

\section{Approximations for aggregate scattering cross section}

This part begins with a short summary on the exact methods. It is followed by the study of the relation between aggregate scattering cross section and their physical characteristics. Finally, four approximations are described and an illustration in the field of precipitation is presented.

\subsection{Exact theory for non spherical particles and aggregates}

The presented summary (see Table 1) of the different exact methods is not exhaustive. But we try to show several methods used to calculate the optical properties of an aggregate. We invite the reader to consult the article of Kahnert (2003) to have a more complete range of these methods and the papers series of Mishchenko et al. (2007, 2008). It is rather difficult to classify them precisely and especially to enumerate all of them.

Nevertheless one can classify them in three main categories:

1. Methods based on the partial derivative equations which calculate the scattering field by solving the Maxwell equations or the Helmholtz equation. They are subjected to the boundary conditions suitable in the time or the frequency domain.

2. Methods based on integration over volume or surface of equations derived from the Maxwell equations. Thus, the boundary conditions are automatically included in the solution. 3. The other methods are known as hybrids since they derive from the various approaches. 


\begin{tabular}{|c|c|c|c|}
\hline Name & Principle & Applications & Strong and weak points \\
\hline \multicolumn{4}{|c|}{ Methods based on partial derivative equations } \\
\hline $\begin{array}{l}\text { S.V.M. } \\
\text { Separation } \\
\text { of Variable } \\
\text { Method }\end{array}$ & $\begin{array}{l}\text { Method applied in the case of } \\
\text { the Mie theory; it can be } \\
\text { applied when the boundary of } \\
\text { the considered particle } \\
\text { coincides with the reference } \\
\text { frame. }\end{array}$ & $\begin{array}{l}\text { In any reference frame, where } \\
\text { the variables separation can be } \\
\text { applied. Asano and Yamamoto } \\
\text { (1979) used this technique to } \\
\text { determine the optical properties } \\
\text { of a spheroid. }\end{array}$ & $\begin{array}{l}\text { - The solution obtained is } \\
\text { known as exact but } \\
\text { calculations are long. } \\
\text { - the operation for each } \\
\text { orientation must be } \\
\text { repeated } \\
\text { - } \quad \mathrm{N} \approx \mathrm{O}\left(\mathrm{x}^{3}\right)\end{array}$ \\
\hline $\begin{array}{l}\text { F.D.T.D. } \\
\text { Finite } \\
\text { Differences } \\
\text { Time } \\
\text { Domain }\end{array}$ & $\begin{array}{l}\text { This method consists of } \\
\text { discretizing the Maxwell } \\
\text { equations, in space and time. } \\
\text { Then to solve them it is } \\
\text { necessary to start from the } \\
\text { initial values (Yang and Liou, } \\
\text { 2000). }\end{array}$ & All particle shapes. & $\begin{array}{l}\text { - the operation for each } \\
\text { orientation must be } \\
\text { repeated } \\
-\mathrm{N} \approx \mathrm{O}\left(\mathrm{x}^{4}\right)\end{array}$ \\
\hline $\begin{array}{l}\text { F.E.M. } \\
\text { Finite } \\
\text { Element } \\
\text { Method }\end{array}$ & $\begin{array}{l}\text { This method consists in } \\
\text { discretizing the Helmholtz } \\
\text { equation in space and solving } \\
\text { numerically using the boundary } \\
\text { conditions. } \\
\text { (Coccioli et al., 1996). }\end{array}$ & All particle shapes. & $\begin{array}{l}\text { - the operation for each } \\
\text { orientation must be } \\
\text { repeated } \\
\text { - the precision depends } \\
\text { on the grid which must } \\
\text { be selected according to } \\
\text { the particle shapes } \\
\text { - } \mathrm{N} \approx \mathrm{O}\left(\mathrm{x}^{7}\right)\end{array}$ \\
\hline $\begin{array}{l}\text { P.M.M. } \\
\text { Point- } \\
\text { Matching } \\
\text { Method }\end{array}$ & $\begin{array}{l}\text { In this method, the internal and } \\
\text { external fields are expressed as } \\
\text { a spherical harmonic vector. } \\
\text { The tangential field at the } \\
\text { boundary of a particle must be } \\
\text { continuous for a fixed number } \\
\text { of points belonging to the } \\
\text { particle surface. }\end{array}$ & $\begin{array}{l}\text { Normally all particle shapes, } \\
\text { but problems are known for the } \\
\text { elongated geometries. }\end{array}$ & $\begin{array}{l}\text { - This method is limited } \\
\text { to the quasi spherical } \\
\text { particles, it has a dubious } \\
\text { convergence, and thus, } \\
\text { requires a long CPU time } \\
\text { (Wriedt, 1998). }\end{array}$ \\
\hline \multicolumn{4}{|c|}{ Volume or surface integration } \\
\hline $\begin{array}{l}\text { V.I.E.M. } \\
\text { Volume } \\
\text { Integral } \\
\text { Equation } \\
\text { Method }\end{array}$ & $\begin{array}{l}\text { The field inside and outside the } \\
\text { volume is expressed in terms } \\
\text { of incident and internal fields } \\
\text { for the selected volume. } \\
\text { - the internal field is evaluated } \\
\text { by considering, for each volume } \\
\text { element, as being constant: } \\
\text { M.O.M (Method of Moments) } \\
\text { (Harrington, 1968). } \\
\text { Alternatively, } \\
\text { - each element is regarded as a } \\
\text { dipole: D.D.A (Discrete Dipole } \\
\text { Approximation) } \\
\text { (Draine and Flatau, 1994) }\end{array}$ & $\begin{array}{l}\text {-Inhomogenous, anisotropic } \\
\text { particles }\end{array}$ & $\begin{array}{l}\text {-MoM et DDA have a } \\
\text { long CPU time } \\
\text { - the operation for each } \\
\text { orientation must be } \\
\text { repeated } \\
-\mathrm{N} \approx \mathrm{O}\left(\mathrm{x}^{9}\right)\end{array}$ \\
\hline
\end{tabular}

Table 1 : Methods allowing to treat the light scattering by a non spherical particle or an aggregate (N: Number of operations in algorithm (Kahnert, 2003)). 
It is important to specify the meaning of the "T-matrix method" expression which is found in a lot of publications.

In the T-matrix method, the incident and scattering fields are expressed in the form of a series of spherical vector wave functions. This approach is named the T-matrix method, when the expansion coefficients of the incident wave and the scattering wave are connected by a linear transformation ( $\mathrm{T}$ : Transition). This matrix $\mathrm{T}$ contains all the information on the particle's optical properties for a given wavelength. It is a function of the size parameter, the shape, the optical refractive index of the considered particles, but it does not depend on the incident field. Thus this matrix is not to be computed at each particle orientation change or change of the incident wave direction.

To classify the publications set relying on this method, a database was carried out by Mishchenko et al. (2004, 2007, 2008). This method is, in fact, a technique of calculation, which is found associated with various methods (eg.: SVM). Thus, any method making it possible to formulate the problem in the way of a matrix $\mathrm{T}$ is called the T-Matrix method.

The solving by separation of variables (SVM) for only one sphere can be enlarged to an aggregate of spheres by using the translation theorem for the spherical wave vector functions which expresses them in various bases of coordinates, and, by applying a superposition principle. The total scattering field for an aggregate is then represented by the superposition of the individual scattering fields resulting from each particle knowing that these fields are interdependent. Moreover, one can formulate the problem in the way of a T-matrix.

This method is very precise but its computation time depends on the number and the size parameter of primary particles.

We will use in the continuation of this text a method which is in fact a particular case of the T-matrix method (Mishchenko et al., 2004) bearing the name of GMM (Generalized Multiparticle Mie-solution) .

We did not find a comparison of the various methods, except an article of Hovenier et al. (1996) which compared the T-matrix (method by surface integration), SVM and DDA. This article shows that the latter is not completely in agreement with the two other methods. As no study was undertaken in this direction, the work presented in this article is achieved with a method which seems, closest to the one used for a simple sphere and validated by the experiment (Xu and Gustafson, 2001): GMM. The details of GMM are given by Xu (1995, 1996, 1997a,b, 1998a). 


\subsection{Main features of scattering properties of aggregates}

We present a summary of the main features of scattering properties of aggregates. Results are coming from the calculated optical properties of aggregates such as:

- aggregates of spherical primary particles

- number of primary particles in the aggregate $N \in[1,100]$

- primary particle size parameter $(x)$ in the range : $[0.013,9.25]$.

- three different materials $\left(\mathrm{SiO}_{2}, \mathrm{Al}_{2} \mathrm{O}_{3}, \mathrm{TiO}_{2}\right)$; non absorbing materials

Optical properties are calculated by using GMM theory.

First of all it is interesting to study the effect of the inter-particle distance on the scattering cross section.

\subsubsection{Case of a two-sphere set}

The evolution of the scattering cross section of a two-sphere set according to the type of material, their size parameter and the center to center distance has already been studied by Mishchenko et al. (1995) and Quirantes et al. (2001) .

The $K_{X u}$ parameter for different materials was used. $K_{X u}$ is defined as the ratio between the scattering cross section of an aggregate and the sum of scattering cross section of $\mathrm{N}$ primary particles which form this aggregate (so, the denominator assuming non-interacting and noninterfering spheres).

$$
K_{X u}=\frac{C_{X u, N}}{N C_{M i e, 1}}
$$

The two spheres, denoted $i$ and $j$, were gradually separated (centre to centre) by a factor $F_{i j}$ proportional to their diameter, until they do not interact any more ( $K_{X u}$ is equal to 1$)$. As the separation distance is denoted $d_{i j}$, the factor obeys the relation:

$F_{i j}=d_{i j} /(2 a)$

$a$ is the radius of the primary particle. 

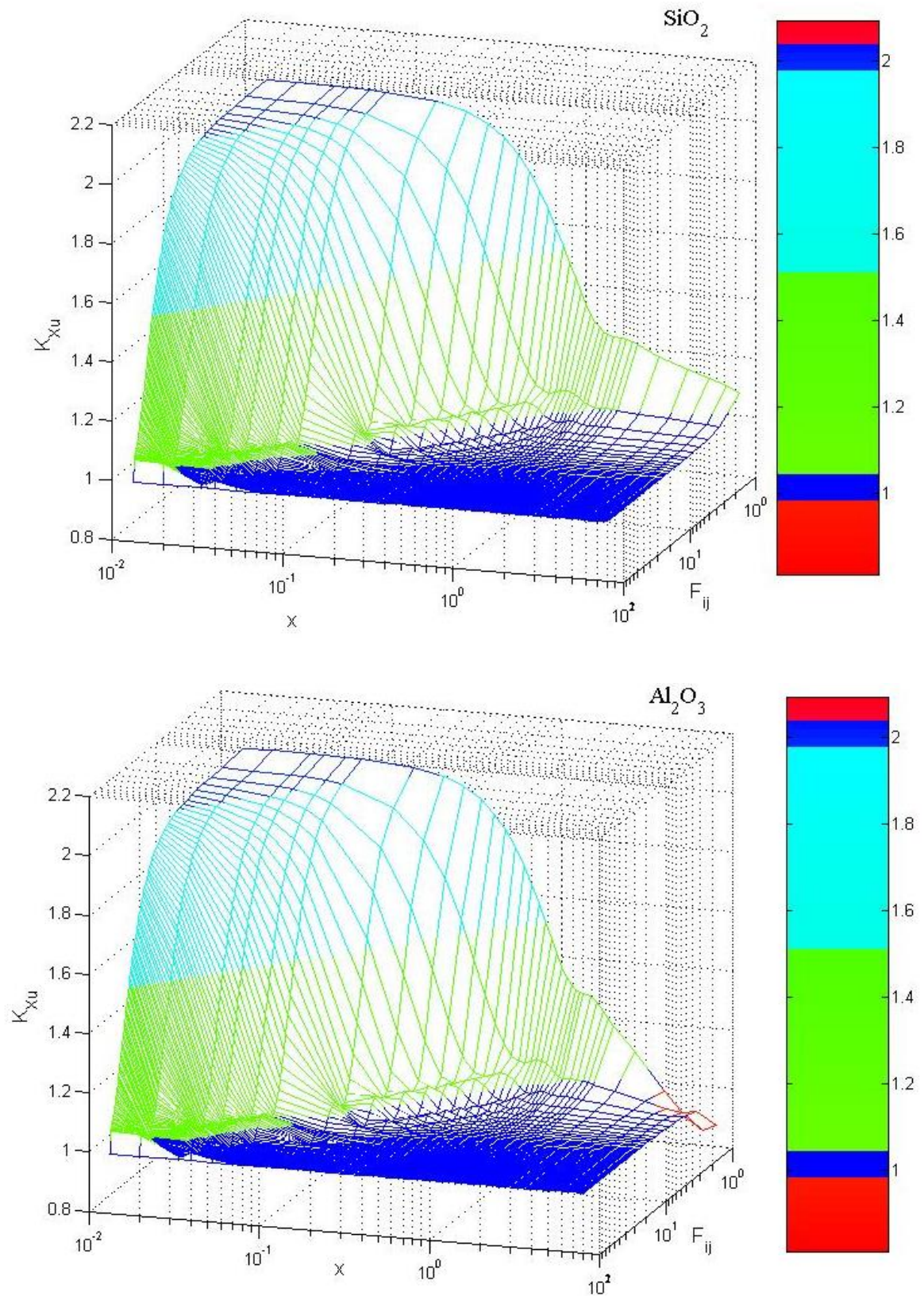


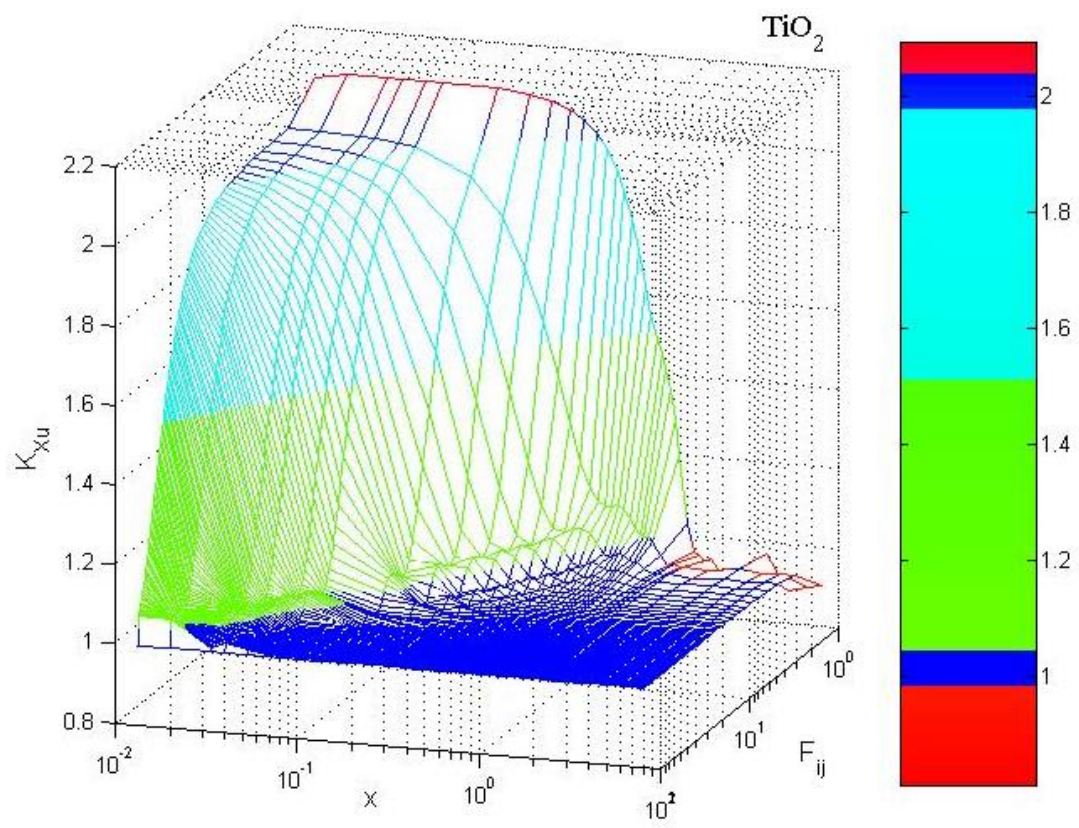

Fig. 6. Evolution of $K_{X u}$ according to the size parameter and the distance factor ranging between $[1 ; 100]$ for the three materials $\left(\mathrm{SiO}_{2}, \mathrm{Al}_{2} \mathrm{O}_{3}, \mathrm{TiO}_{2}\right)$

According to Fig. 6, for size parameter smaller than 0.5, smaller is the primary particle, the distance factor must be greater so that there is no interaction. We thus join the conclusion of Kolokolova and Gustafson (2001): a suspension consisting of Rayleigh scatterers as primary particles has to have a very weak volume fraction to avoid multiple scattering, whatever the relative refractive index.

Interaction between particles cannot be ignored even if $F_{i j}>4$ (for the whole range of the size parameter).

For spheres in contact, $K_{X u}$ (Fig. 6) increases with decreasing size parameter up to a value close to 2. When the two spheres are large enough, the deviation from the non interacting limit is negligible (e.g. $K_{X u}<1.1$ for $x>5$ ).

It thus appears useful to evaluate the critical inter-particle distance for which interaction is negligible. An approximate method for aggregate optics calculation could take it into account.

\subsubsection{Case of aggregate $(\mathrm{N}>2)$}

Auger et al. (2003) studied the relation between extinction cross section of an aggregate, its shape (linear or compact configuration) and the number of primary particles $(2,4,8,13)$ in the case of titania $\mathrm{TiO}_{2}$ (the optical refractive index being equal to 2.8). In this article, the average 
extinction cross section (average on the polarization and the incident wave direction) divided by the aggregate volume (made up of monosized spheres) is calculated, according to their particle radius (between $0.04 \mu \mathrm{m}$ and $0.132 \mu \mathrm{m}$ ). It is found that there exist two size ranges (for $\lambda=0.546 \mu \mathrm{m}$ ). For a primary particle radius smaller than $0.08 \mu \mathrm{m}-0.09 \mu \mathrm{m}$, an isolated primary particle scatters less than if it were contained in an aggregate. Primary particles belonging to the second range behave in an opposite way. They show that there exists two size ranges concerning the effect of aggregate shape: in the first range, a compact configuration scatters more than linear configuration and conversely for the second range. Lastly, a comparison with the equivalent sphere approximation shows that the latter is not suitable. Auger et al (2005) and Jacquier and Gruy (2007a) perform similar studies in the way that they compare the scattering cross section for various configurations. Auger et al. (2005) study is based on distribution of randomly generated aggregates by classical mechanisms of aggregation.

Jacquier (2006) and Jacquier and Gruy (2007a) enlarged the study using different optical refractive indices, a greater range of primary particle size parameter, and different configurations. They noted the effect of the primary particle number and the aggregate morphology. The results are summarized in the two next paragraphs.

- Effect of the number of primary particles on the scattering cross section

In the paper of Jacquier and Gruy (2007a), two extreme configurations were compared (linear and compact configuration). For each configuration, Jacquier and Gruy noticed that there exist two ranges. The first one is for $K_{X u}$ larger than 2, and the other one for $K_{X u}$ ranging between 0 and 2 . The value of the size parameter of the primary particles corresponding to the range boundary is a weak function of the optical refractive index, the primary particle number and the configuration. However, the authors suggest the first range for $x \in[0,2]$ (Fig.7a) and the other one for $x \in[2,10]$ (Fig.7b) (the limit of their study is for a size parameter smaller than 10). 


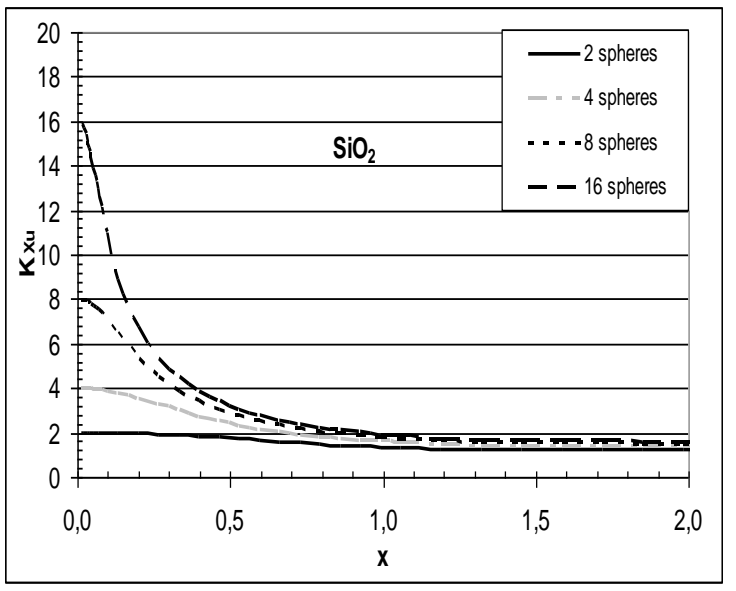

a

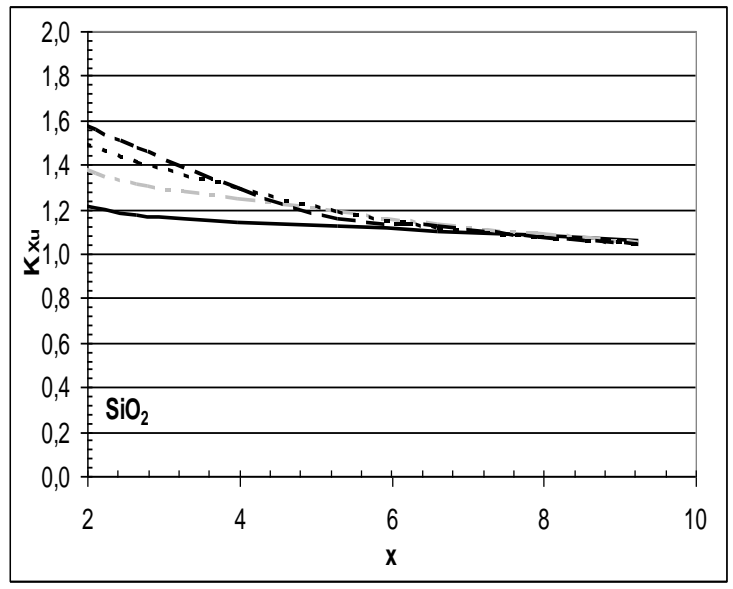

b

Fig.7: $K_{X u}$ as a function of the size parameter for the linear configuration with $\mathrm{N}$ primary particles $(2,4,8,16)$ and for $\mathrm{SiO}_{2}($ Jacquier and Gruy, 2007a): (a) for the range $x \in[0,2]$ and (b) $x \in[2,10]$.

They conclude (as shown in Fig.7):

- for $x \in[0,2]$, larger is the number of primary particles, larger is the scattering cross section whatever the configuration. Indeed, in the case of very small size parameter, the aggregate scattering cross section is proportional to the particle number square and to the primary particle scattering cross section $\left(C_{X u, N} \propto N^{2} C_{M i e, 1}\right)$. This relation is checked on a lesser scale by aggregates with high refractive index (e.g., $\mathrm{TiO}_{2}$ ). In addition the decrease of $K_{X u}(x)$ seems to depend on the configuration.

- for $x \in[2,10], K_{X u}(x)$ is not yet equal to 1 (Fig. 7b), i.e. the aggregate scattering cross section is not the sum of scattering cross sections of each primary particle.

\section{- Effect of the aggregate morphology on scattering cross section}

As illustrated in Fig.8, it is possible to establish a classification of the configurations according to their scattering cross section. In the $x$-domain, where $K_{X u}>1$, the scattering cross section of the compact configuration is higher than that of the plane configuration, itself higher than that of $\mathrm{p} 1$ and $\mathrm{p} 2$ configurations (which are very close, Fig.9). The linear configuration is the weakest. The order is reversed for the other $x$-domain $\left(K_{X u}<1\right)$. Thus, there are two extreme configurations: linear and compact between which other configurations are. 


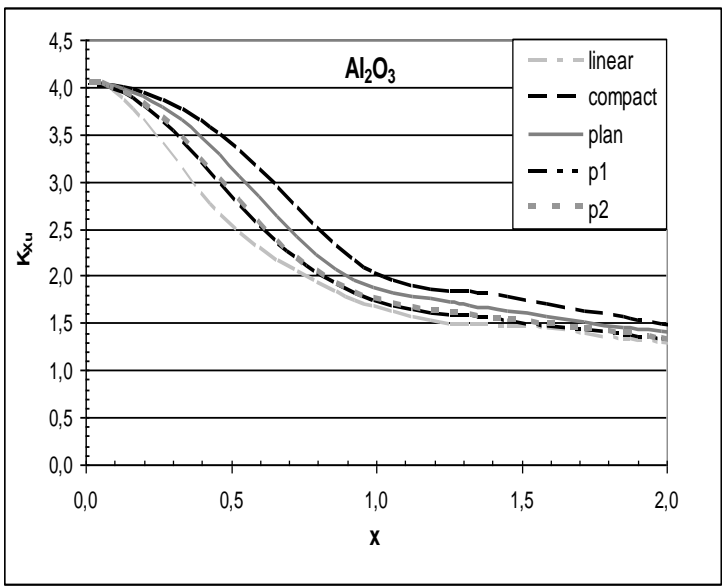

a

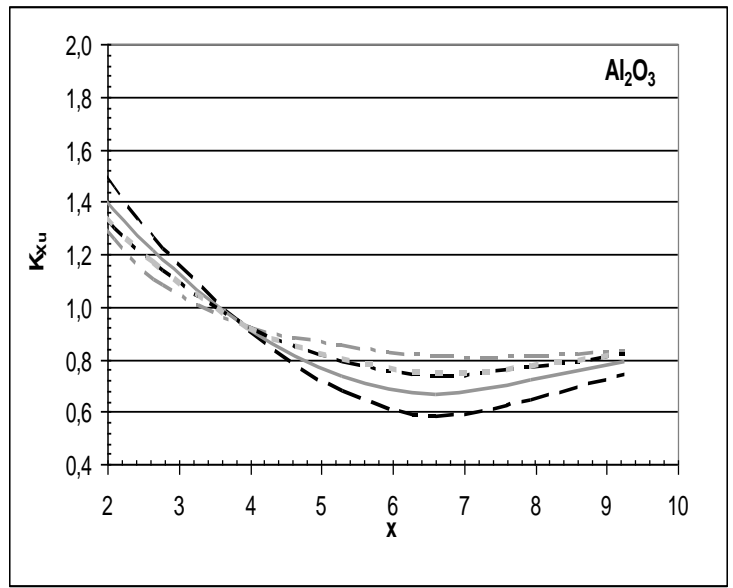

b

Fig.8: $K_{X u}$ as a function of the size parameter for aggregates with four primary particles (linear, compact, plan, p1, p2) for $\mathrm{Al}_{2} \mathrm{O}_{3}$

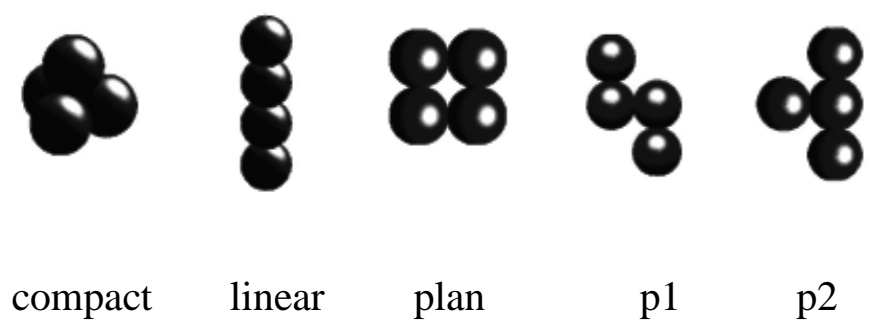

Fig.9: Different aggregate configurations in the case of four primary particles.

The primary particles arrangement, i.e. the aggregate configuration, is not without effect on the scattering cross section, nevertheless, the number of primary particles in it is the prevailing parameter in the range $x \in[0 ; 2]$. In the second range $(x \in[2 ; 10])$, the configuration is more important than the number of primary particles.

\subsubsection{Conclusion on aggregate scattering cross section}

The study of scattering $\left(\mathrm{C}_{\mathrm{sca}}\right)$ cross sections of aggregates obtained with the exact method revealed that:

- the distance between particles is a relevant parameter for $\mathrm{C}_{\text {sca }}$

- different aggregate configurations, following its shape or the number of primary particles which it contains, are perfectly distinguishable,

- the number of primary particles is the relevant parameter in the case of small size parameter $x\left(x \rightarrow 0, C_{X u, N} \propto N^{2} C_{M i e, 1}\right)$ 
- there exists, for an aggregate with a given number of primary particles, two extreme configurations (linear and compact) between which the cross sections of the others are located.

\subsection{Approximate methods (CS, BPK, AD, ERI) for aggregates}

In this sub-section, we describe different approximate methods: Compact Sphere method (CS), Berry-Percival-Khlebtsov method (BPK), Anomalous Diffraction method (AD), Effective Refractive Index method (ERI). A first comparison between these four methods was published by Gruy (2001) in connexion with aggregation of $\mathrm{SiO}_{2}$ micro-particles in water.

The study of the parameters influencing the optical properties of aggregate began with Fuller and Kattawar (1988, a and b). Rouleau (1996) compared several approximate methods for optical properties based on:

- RGD approximation

- Non interacting spheres

- Equivalent volume sphere

- Equivalent projected area sphere

This study was carried out only for compact aggregates with 30 primary particles whose size parameter was smaller than 0.6 and the refractive index was kept constant $(m=1.7+0.1 i)$. He concluded that the above-mentioned methods are not efficient except the one using the projected area.

The differences between the methods quoted in the next paragraphs are evaluated in Table 2 . We chose to differentiate porosity and arrangement. The validity range of all these methods is normally the whole size parameter range except for $\mathrm{AD}$, which, as already mentioned in subsection 3.3, is to be used only in the case of large particles.

\begin{tabular}{|c|c|c|c|}
\hline \multirow{2}{*}{ Method } & \multicolumn{2}{|c|}{ Does it take into account ? } & Does it use: \\
\cline { 2 - 3 } & *the arrangement & *the interactions & $\begin{array}{c}\text { Maxwell-Garnett equation? } \\
\text { (porosity) }\end{array}$ \\
\hline CS & no & no & no \\
\hline ERI & yes & no & yes \\
\hline PBK & yes & yes & no \\
\hline AD & yes & yes & no \\
\hline
\end{tabular}

Table 2: Comparison of approximate methods 
In the next paragraphs, scattering cross section values from approximate method $\left(C_{\text {method,N }}\right)$ and exact method ( $C_{X u, N}$ ) will be compared. Then, the ratio $K_{m}$ is defined as:

$$
K_{m}=\frac{C_{m e t h o d, N}}{C_{X u, N}}
$$

\subsubsection{Compact Sphere (CS)}

This method has to be mentioned because it is used as the first coarse approximation by investigators and by particle sizer manufacturers. One finds it under the name of equivalent sphere (in volume), and it will be compared with the other methods.

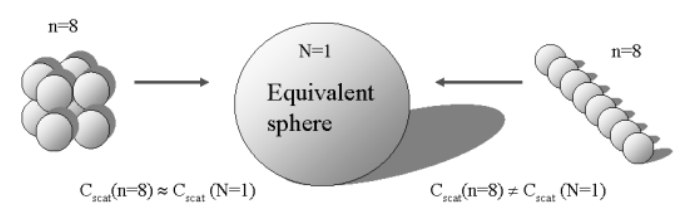

Fig.10: Compact Sphere method

The aggregate is regarded as a full sphere, i.e. containing all the matter. This method can be valid for aggregates of high compactness. The scattering cross section $C_{C S, N}$ is then evaluated with the theory of Mie.

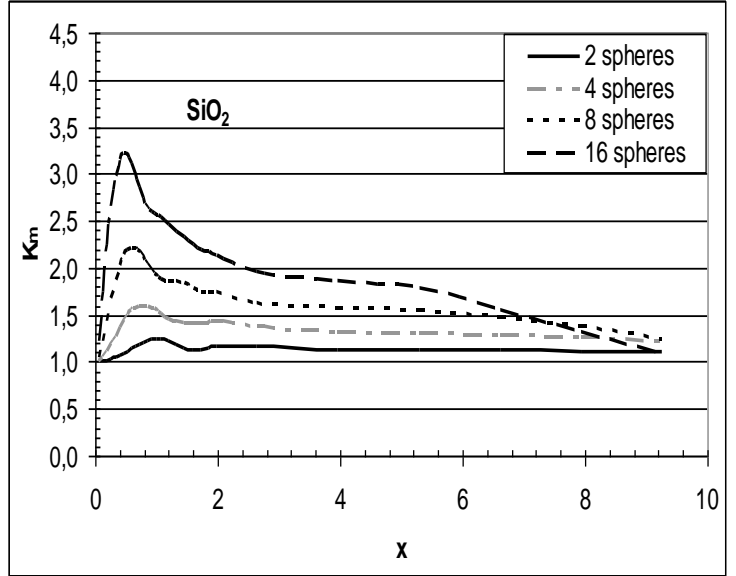

a

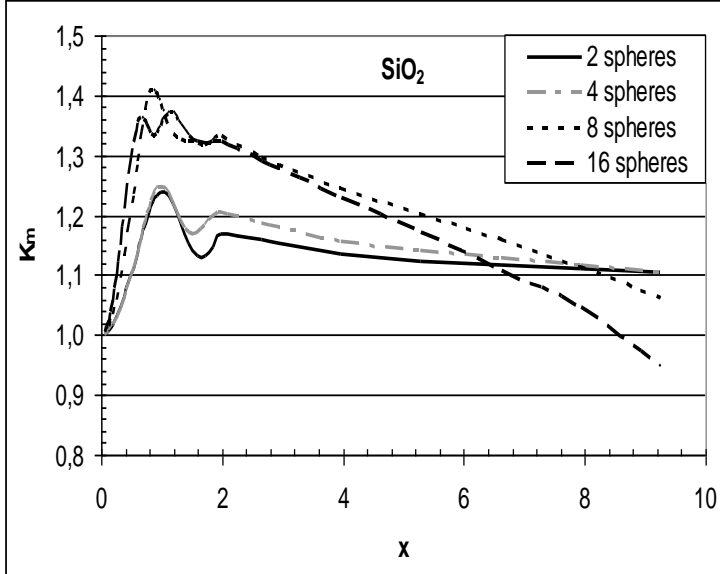

b

Fig.11: compact sphere approximation: $K_{m}$ as function of the size parameter for $\mathrm{SiO}_{2}$ (a) linear configuration, (b) compact configuration

As shown in the Fig. 11, compact sphere method overestimates the scattering cross section for $x<7$, whatever the configuration. As we will see in paragraph 4.3.3, an aggregate can be considered as a (porous) sphere with an effective refractive index. Whatever the chosen 
equivalent sphere, the value of the scattering cross section calculated by Mie theory is always smaller than the one obtained from CS method. Moreover, this method does not take into account the interactions (interference and interaction).

\subsubsection{Berry-Percival-Khlebtsov (BPK)}

This method originates from the work of Berry and Percival (1986) and Khlebtsov (1996). The three following points constitute the stages of BPK method:

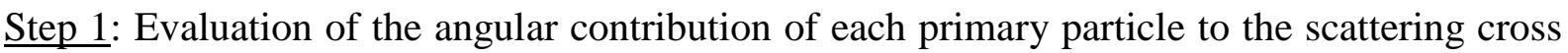
section:

$F_{1}(\theta)=\frac{C_{M i e, 1}}{C_{R G D, 1}} F(\theta)$

where $F(\theta)$ is coming from the RGD approximation for a sphere (see Eq. $8 \mathrm{~b}$ ). $F_{1}(\theta)$ is the corrected function for $F(\theta)$ in order to verify: $\int_{0}^{\pi} F_{1}(\theta) d \theta=C_{M i e, 1}$

Step 2: Calculation of the interferences of scattering waves for each pair of primary particles leading to a structure factor $S$ which does not depend on polarization. The structure factor is related to the aggregate morphology through the inter-particle distances.

$S(\theta)=\left[N+\sum_{i=j=1, i \neq j}^{N} R_{i j}(\theta)\right] / N^{2}$

where

$$
R_{i j}(\theta)=\frac{\sin \left(2 k d_{i j} \sin \left(\frac{\theta}{2}\right)\right)}{2 k d_{i j} \sin \left(\frac{\theta}{2}\right)}
$$

and $d_{i j}$ is the distance between $i$ and $j$ particles.

Step 3: Use of a corrective coefficient $d$ taking into account the multiple scattering (Berry and Percival, 1986) 
$d^{-1}=\left[1-\frac{3}{2 N}\left(-a_{i 1} p_{r}-a_{r 1} p_{i}\right)\right]^{2}+\left[\frac{3}{2 N}\left(a_{r 1} p_{r}-a_{i 1} p_{i}\right)\right]^{2}$

with

$a_{r 1}$ et $a_{i 1}$ are the real and imaginary parts of the first Mie coefficient $a_{1}$

$$
\begin{aligned}
& p_{r}=2 \sum_{j>i, i=1}^{N} p_{r_{1}}\left(k d_{i j}\right) \\
& p_{i}=2 \sum_{j>i, i=1}^{N} p_{i_{1}}\left(k d_{i j}\right) \\
& p_{r_{1}}(x)=\left(\cos x f_{1}(x)-\sin x f_{2}(x)\right) / x^{2} \\
& p_{i_{1}}(x)=\left(\sin x f_{1}(x)+\cos x f_{2}(x)\right) / x^{2} \\
& f_{1}(x)=\sin x-\frac{x}{3} f(x)+\frac{1}{x}\left(f(x)-\frac{\sin x}{x}\right) \\
& f_{2}(x)=\frac{\sin x}{x}-f(x) \\
& f(x)=\frac{3}{x^{3}}(\sin x-x \cos x)
\end{aligned}
$$

\section{Conclusion of steps:}

$$
C_{B P K, N}=N^{2} d \int_{0}^{\pi} F_{1}(\theta) S(\theta) d \theta
$$

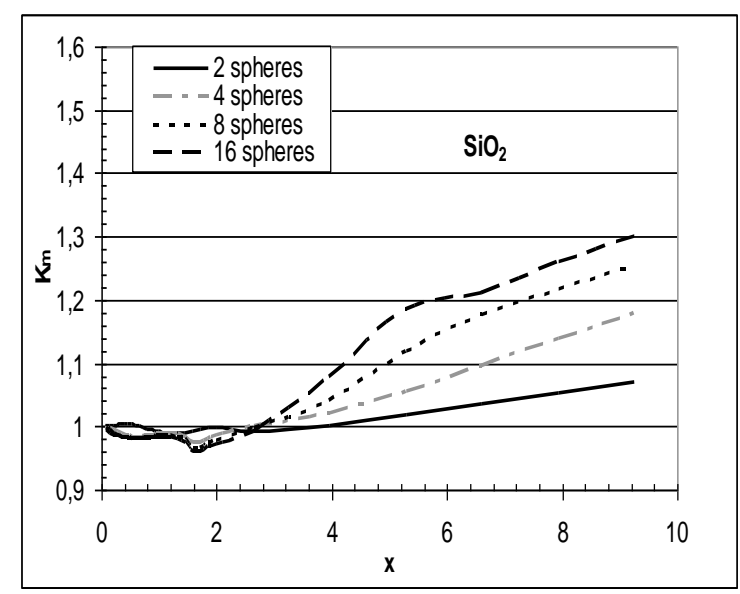

a

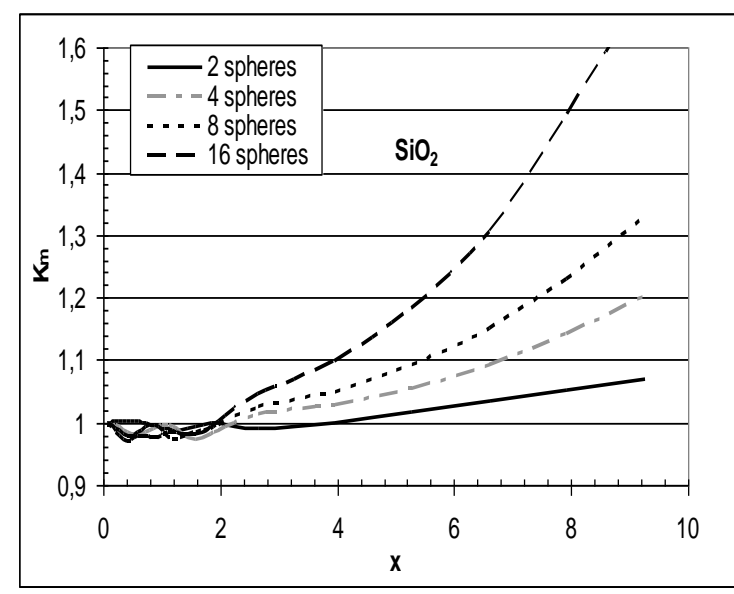

b 
Fig.12: BPK approximation: $K_{m}$ as function of the size parameter for $\mathrm{SiO}_{2}$ (a) linear configuration, (b) compact configuration

Figure 12 shows that BPK method is a satisfactory approximation of the exact method for the small size parameter $(x<2)$. The BPK method leads to an error smaller than $10 \%$ for a size parameter ranging between 0 and 2 in the case of $\mathrm{SiO}_{2}$ and of $\mathrm{Al}_{2} \mathrm{O}_{3}$. For higher refractive index (i.e $\mathrm{TiO}_{2}$ ), the error increases until it reaches $30 \%$ (for more restricted size parameter range $[0 ; 1])$. BPK method shows that the pair interactions must be taken into account only for small size parameters; their contribution in scattering cross section calculation is less in the case of large aggregated primary particles.

\subsubsection{Effective refractive index}

We have shown in paragraph 4.2.2, that the location of the primary particles inside an aggregate and its shape had an effect on the scattering cross section. The effective refractive index (ERI) method considers the shape. Knowing that the projected area of the scattering body (on the plane ( $\vec{E}, \vec{H})$ of the incident wave) is a relevant parameter in optics, we consider an equivalent sphere starting from the aggregate projected area (Fig. 13).

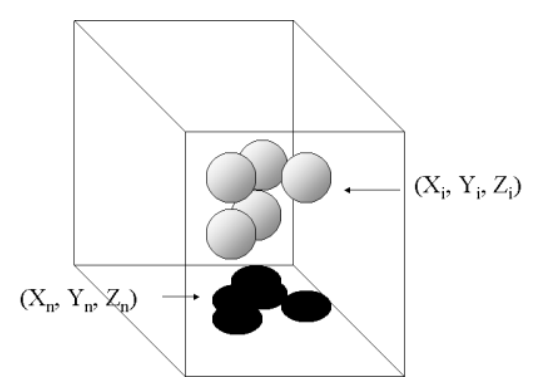

Fig.13. Projected area representation

Projection is carried out according to several successive planes (plane perpendicular to the incident wave vector). This corresponds to random rotation that takes place in a real situation (for instance, aggregate in a turbulent flow). Then, an average projected area $\left\langle S_{p}\right\rangle_{O}$ is calculated and an equivalent projected area sphere is defined. Successively, it can be deduced, the equivalent radius $a_{\mathrm{N}, \mathrm{e}}$, the solid volume fraction inside the sphere $\bar{\phi}_{a}=\left(N a_{1}^{3}\right) / a_{N, e}^{3}$, the effective refractive index $m_{a}$ using the Maxwell-Garnett theory $\frac{m_{a}^{2}-1}{m_{a}^{2}+2}=\bar{\phi}_{a} \frac{m^{2}-1}{m^{2}+2}$, and then the extinction cross section $C_{E R I, N}$ by means of Mie theory. 


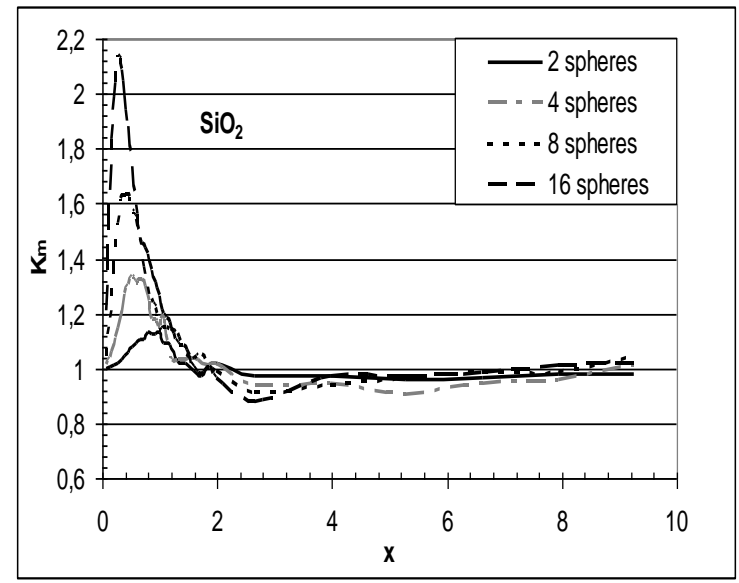

a

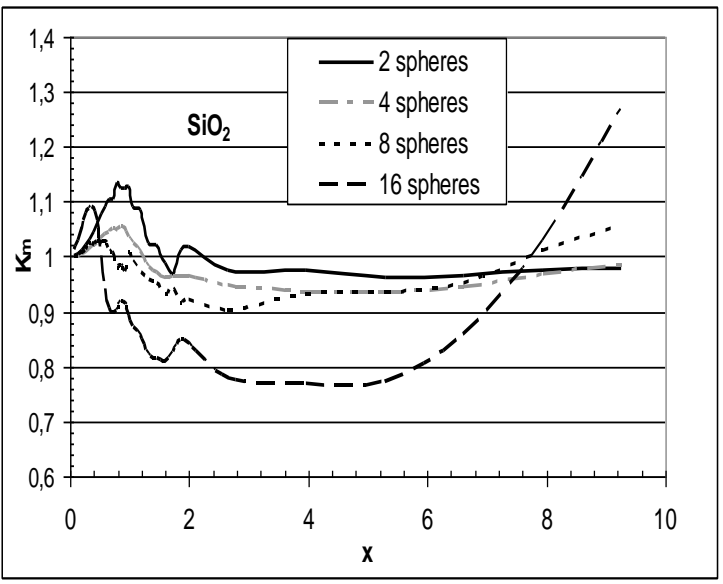

b

Fig.14: ERI approximation: $K_{m}$ as function of the size parameter for $\mathrm{SiO}_{2}$ (a) linear configuration, (b) compact configuration

This method is more efficient than the other equivalent sphere methods, because the solid volume fraction in this sphere is always high $\left(0.1<\bar{\phi}_{a}<1\right)$.

ERI method behaves differently according to the configuration for small size parameter (Fig. 14). Indeed, $C_{\text {sca }}$ value calculated with this method is higher than the scattering cross section calculated with the exact method for a linear configuration (Fig. 14a). This deviation can be taken in consideration and calculation has to be corrected in order to reduce the deviation between ERI and exact methods.

Jacquier and Gruy $(2007 \mathrm{a}, \mathrm{b})$ proposed a corrective factor $F\left(x, d_{1} / a\right)$ for the scattering cross section $C_{E R I, N}$. This is written as:

$C_{E R I, N}^{c o r r}=C_{E R I, N} / F\left(x, d_{1} / a\right)$

Where $d_{l}$ is a morphological parameter defined as:

$d_{1}=\frac{1}{N(N-1)} \sum_{i, j} d_{i j}$

\subsubsection{Anomalous Diffraction (AD)}

In paragraph 4.2.2 it has been already mentioned that morphology becomes more important for large size parameters. The anomalous diffraction approximation, clarified in sub-section 3.3 (Van de Hulst, 1981), accounts for the aggregate morphology by means of the intercept (chord) of a light ray and the aggregate (Fig. 15). 


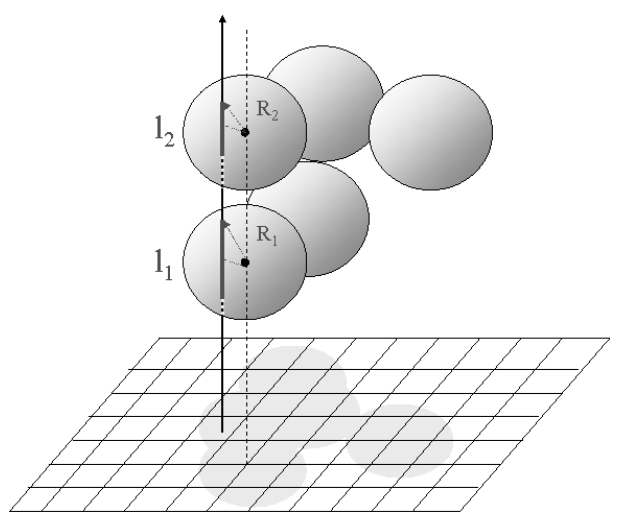

Fig.15. definition of a chord

The various possible chords $l_{1}, l_{2} \ldots$ were evaluated and introduced as $l=\sum_{i} l_{i}$ into Eq.19.

$C_{A D, N}=2 \iint_{\left[S_{p}\right]}\left(1-\cos \frac{2 \pi}{\lambda} l(m-1)\right) d y d z=2 \iint_{\left[S_{p}\right]}(1-\cos x(l / a)(m-1)) d y d z$

$\left[\mathrm{S}_{\mathrm{p}}\right]$ is the projection plane. Details or examples on expressions relating $l / a$ and $(y, z)$ can be found in Yang et al. (2004) and Gruy and Jacquier (2008).

This calculation is repeated whilst rotating the aggregate or changing the projection plane. So, a mean value of scattering cross section is deduced.

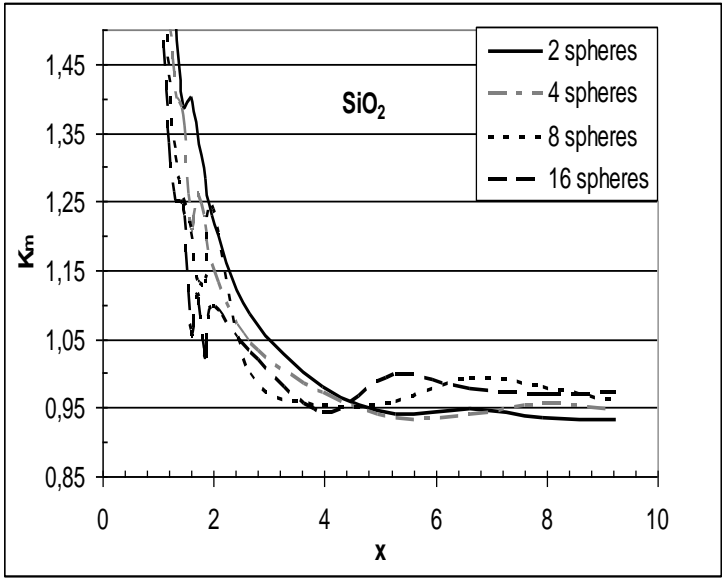

a

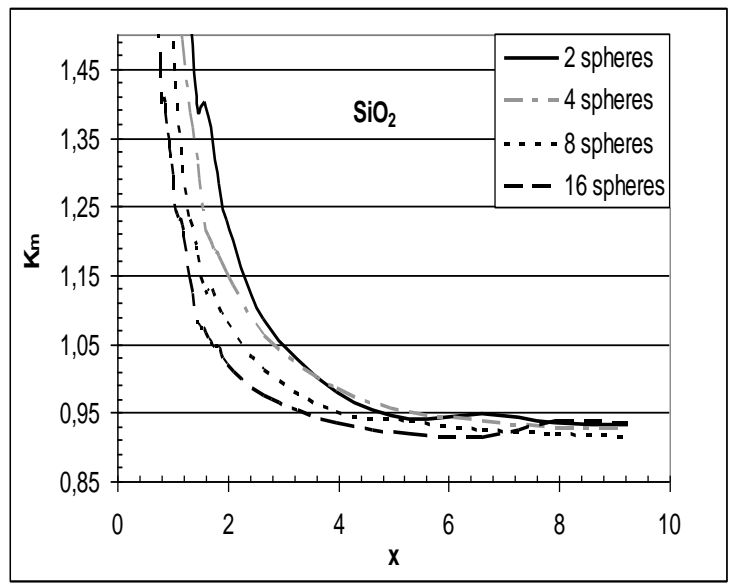

b

Fig.16: $\mathrm{AD}$ approximation: $K_{m}$ as function of the size parameter for $\mathrm{SiO}_{2}$ (a) linear configuration, (b) compact configuration 
As expected, the AD method is not suitable for small size parameters but proves to be a good approximation for a size parameter higher than 2. It is important to recall that $\mathrm{AD}$ is strongly related to the configuration (morphology) since it includes in its formulation the chord length distribution of the aggregate.

\subsubsection{Summary}

Approximate methods facilitate the estimation of the aggregate scattering cross section in a short computation time. Jacquier and Gruy (2007a) evaluated the performance of these four approximate methods with respect to the exact method:

- Methods replacing an aggregate by a compact sphere (CS) are inappropriate.

- BPK (Berry-Percival-Khlebtsov) method is valid for $0<x<2$ with an error which increases with the material refractive index.

- The corrected ERI (Effective Refractive Index) method is the approximate method being able to be efficient on the whole size parameters. The error for a scattering cross section is always smaller than $5 \%$.

- the AD (Anomalous Diffraction) method works fairly well for $2<x<10$ and is less sensitive to the refractive index variation.

\subsection{Application: turbidity versus time during agglomeration process}

As mentioned in section 2, nucleation and growth lead to (primary) particles with a size between $0.1 \mu \mathrm{m}$ and $10 \mu \mathrm{m}$. Then, these particles collide and agglomerate by Brownian motion and/or local shear. So, let us consider agglomeration of small monosized primary particles in a homogeneous suspension. Agglomeration proceeds as a bimolecular reaction, the kinetic constant of which can be expressed in terms of known quantities. Generally, the kinetic constant is a function of sizes of the two colliding particles. However, in the case of Brownian agglomeration or shear agglomeration (but not for shear aggregation, i.e without consolidation of the particle cluster), the kinetic constant $K_{a g}$ weakly depends on the particle size, so that we may consider it as not dependent on particle size. Following Kruyt (1952), modelling of agglomeration with constant $K_{a g}$ leads to simple expressions for number concentrations in agglomerate: 
$N_{j}=N_{0}\left(t / t_{c}\right)^{j-1}\left(1+t / t_{c}\right)^{-j-1}$

$N_{j}$ is the number concentration in agglomerate consisting of $\mathrm{j}$ primary particles. $N_{O}$ is the number concentration in primary particles at time $t=0$. There is no agglomerate at $t=0 . t_{c}$ is the characteristic time of agglomeration. It obeys:

$t_{c}=2 /\left(K_{a g} N_{0}\right)$

For instance, $t_{c}=3 \mu /\left(4 k T N_{0} \alpha\right)$ for Brownian agglomeration. $T, k$ and $\mu$ are the temperature, the Boltzmann constant and the dynamic viscosity, respectively. $\alpha$ is a non dimensional parameter representing the agglomeration efficiency $(0<\alpha<1)$. For sake of simplicity, we consider this parameter as a constant throughout the agglomeration process.

It will be pointed out that $t_{c}$ and then $N_{j}$ do not depend on the agglomerate morphology. The previous expressions are approximate, but are considered as a first and realistic approach of agglomeration dynamics.

At a given time, the turbidity of the suspension contains the contribution of each $\mathrm{j}$ agglomerate:

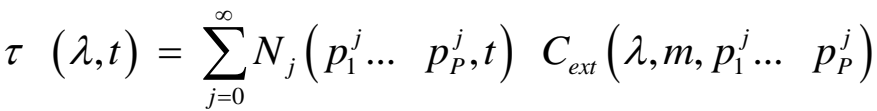

Following ERI method, the internal coordinates relevant for scattering cross section are $a$ (the primary particle radius), $j,\left\langle S_{p}\right\rangle_{O}$. Even if the characteristic time does not depend on the morphology, it appears that large agglomerates have a fractal-like structure. Depending on the agglomeration mechanism, simulations give values of fractal dimension between 1.8 and 2.6. Due to restructuring of agglomerates, the fractal dimension is larger than 2. As the fractal dimension is larger than 2, outer radius of agglomerate is equal to the radius $a_{S, j}$ of the "projected area" equivalent sphere. Small agglomerates do not have the fractal-like structure. However, we have shown (Gruy, 2001) that they can be described by means of a power law relating $a_{S, j}$ and $j$ :

$$
\frac{a_{S, j}}{a}=\left(\frac{\left\langle S_{p}\right\rangle_{O}}{\pi a^{2}}\right)^{1 / 2}=\left(\frac{j}{S_{r}}\right)^{1 / D_{F}}
$$


Later on, we will consider the equation 22 suitable for a wide range of primary particle numbers.

Then,

$\tau(\lambda, t)=\sum_{j=0}^{\infty} N_{j}\left(t / t_{c}\right) C_{e x t}\left(\lambda, m, j, a, D_{F}\right)$

Figures 17 and 18 represent the change of turbidity (normalized by $\tau_{0}(\lambda)=N_{0} C_{e x t}(\lambda, m, a)$ ) with time (normalized by $t_{c}$ ) for agglomeration of silica $(m=1.08)$. Figures 17 and 18 show the effect of two fractal dimensions $\left(D_{F}=2.1 ; D_{F}=2.5\right)$ and two primary particle radii $(a=0.1 \mu \mathrm{m} ; a=1 \mu \mathrm{m})$ respectively at $\lambda=0.4 \mu \mathrm{m}$ and $\lambda=0.8 \mu \mathrm{m}$.

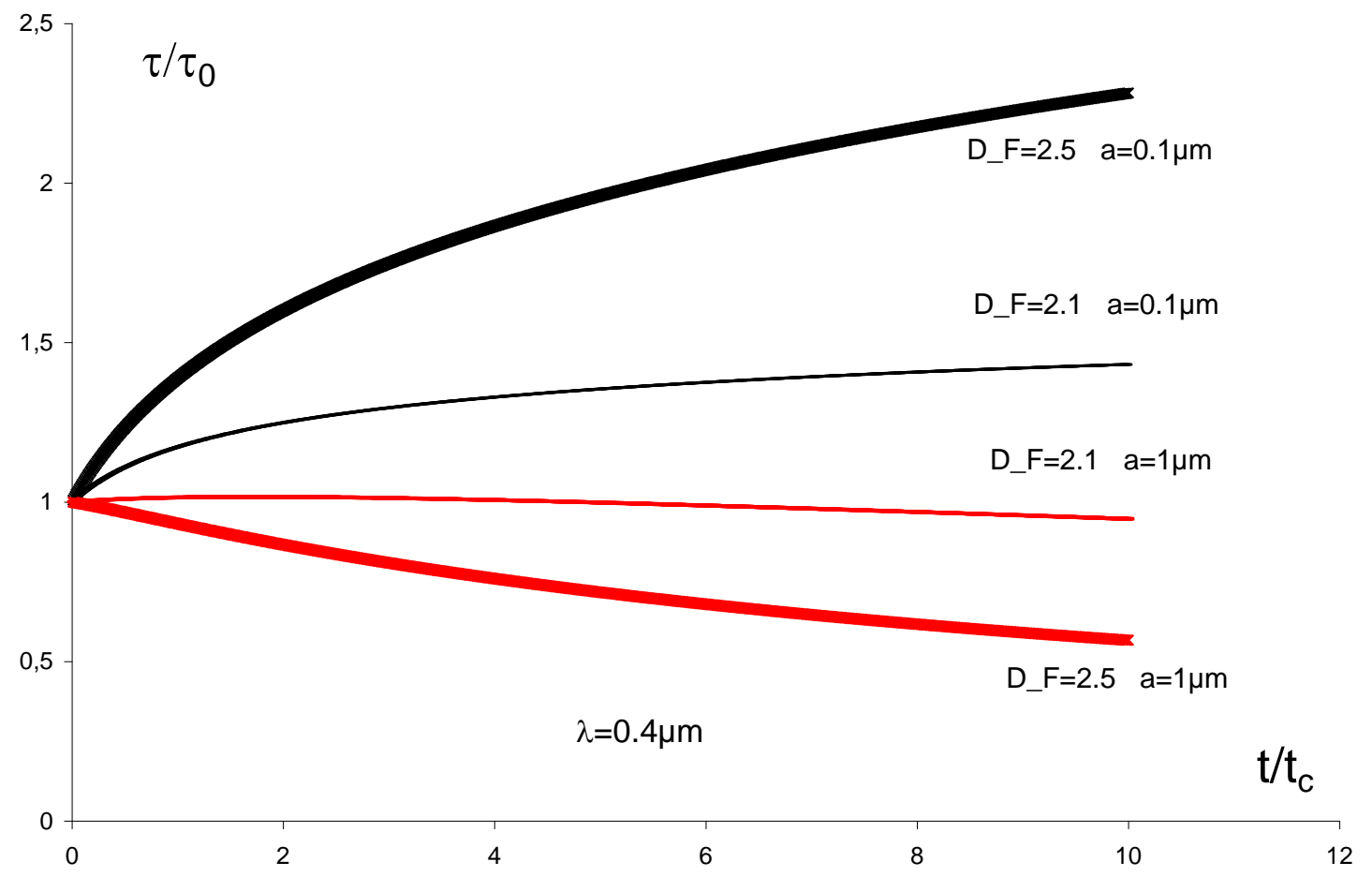

Fig. 17: Normalized turbidity versus time; agglomeration of silica in water; $\lambda=0.4 \mu \mathrm{m}$ 


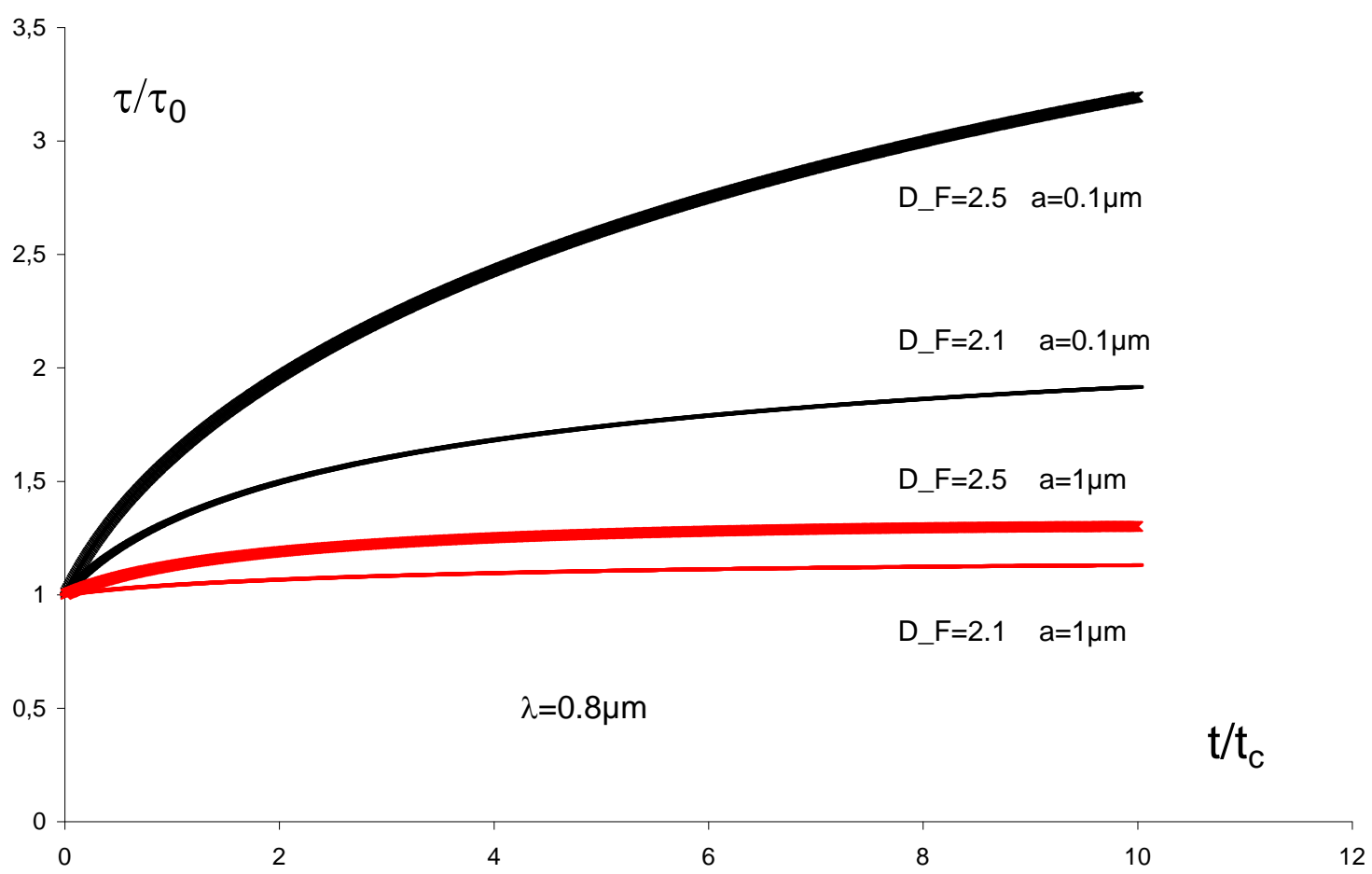

Fig. 18: Normalized turbidity versus time; agglomeration of silica in water; $\lambda=0.8 \mu \mathrm{m}$

\section{Approximation for radiation pressure cross section}

\subsection{Introduction}

Often, precipitation leads to concentrated suspensions. On one hand, transmitted light intensity becomes very weak making backscattering sensors more suitable than turbidimetric ones. On the other hand, multiple scattering takes place. Whatever the considered signal (backscattered, side scattered or transmitted light), the interpretation has to account for multiple scattering. The most popular theory which considers this phenomenon is the radiative transfer theory, particularly its diffusion approximation (Ishimaru, 1978). The relevant phenomena associated with backscattering measurement, are either coherent or incoherent ones. The first one results from interference caused by the double passage of the wave through the same particle (Tsang and Ishimaru, 1984, 1985; Wolf et al., 1988; Akkermans et al., 1988; de Wolf, 1991; Helfenstein et al., 1997). The angular width of the measured intensity peak is proportional to the transport mean free path $L_{t r}=\left(N C_{p r}\right)^{-1}$, where $N$ is the particle number concentration and $C_{p r}$ is the radiation pressure cross section. The second one only considers the multiple scattering: scattered light intensity is also a function of the 
transport mean free path. Theoretical calculations were compared to on- (off-) line experimental data for transmittance (Ishimaru et al., 1983) and retroreflectance (Kuga, 1984; Nichols et al., 1997) experiments with suspension of spherical beads, and a fairly good agreement between both was found.

The radiation pressure cross section is expressed as a function of the extinction and scattering cross sections, and also the asymmetry parameter $(g=\langle\cos \theta\rangle)$ :

$C_{p r}=C_{e x t}-C_{s c a} \cdot g$

In the case of non absorbing material:

$C_{p r}=C_{s c a}(1-g)$

The asymmetry parameter for spheres has been calculated and analytical or empirical expressions have been derived in the framework of different approximations, e.g. geometrical optics and RGD approximations. Empirical expressions for large randomly oriented nonspherical particles were also proposed (see Kokhanovsky, 2001). Rigorous calculations were achieved by different authors (see, for instance, Xu, 1998b).

Up to now, calculations of radiation pressure cross section for aggregates were mainly motivated by calculation of forces acting on interstellar dust illuminated by stars (Kimura and Mann, 1998; Kimura et al., 2002; Iati et al., 2004). Radiation pressure plays a key role in the dynamical behaviour of submicrometer-size grains in the stellar radiation and gravitational fields. Kimura and Mann (1998) studied aggregates composed of 256 primary particles, the radius of which is $0.01 \mu \mathrm{m}$ and that are illuminated by visible light and infrared. The considered materials are silicate and amorphous carbon as representatives of weakly and strongly absorbing materials, respectively. Calculations for randomly oriented fractal-like aggregates (with $D_{F}=2$ and $D_{F}=3$ ) were performed by means of DDA method. Authors showed that the asymmetry parameter smoothly increases with increasing size parameter $x$ (decreasing wavelength) of the primary particle and increases as the fractal dimension decreases if $x<0.16$ (for $x>0.16, g \square 0.7$ ). Asymmetry parameter for aggregates is higher than for volume-equivalent spheres, irrespective of the constituent material. The authors point out that aggregates with small fractal dimension present a large fluctuation in $g$ for different aggregate orientations. Kimura et al. (2002) extended the previous study to larger aggregates $(N<2048)$. They compare radiation pressure cross sections calculated from DDA method, Mie 
theory applied to volume-equivalent sphere (CS method) and Mie theory combined with Bruggeman mixing rule. CS is a rough approximation for the two materials and two fractal dimensions. Mie/Bruggeman approximation is a good approximation for compact aggregates, but performs weakly for loose aggregates especially with non absorbing primary particles. The authors showed that $C_{p r}$ is less dependent on the porosity of aggregates while the values strongly vary with the material composition. Iati et al. (2004) computed, through T-matrix method, optical properties of cosmic dust grains. Grains are aggregates consisting of 31 nonidentical spheres. Materials are also silicate and amorphous carbon. Primary particle size distribution is assumed to be Gaussian-like. The radius of the volume-equivalent sphere is equal to $0.14 \mu \mathrm{m}$. For both materials, aggregation leads to a sharp increase in the extinction and radiation pressure cross sections. The sub-sections 5.2 and 5.3 are respectively devoted to the main features of $C_{p r}$ for aggregates and approximate methods for estimating $C_{p r}$.

\subsection{Main features of radiation pressure cross section}

\subsubsection{Single sphere}

The variation of the asymmetry parameter is presented for spherical particles of various chemical compositions in Fig.19.

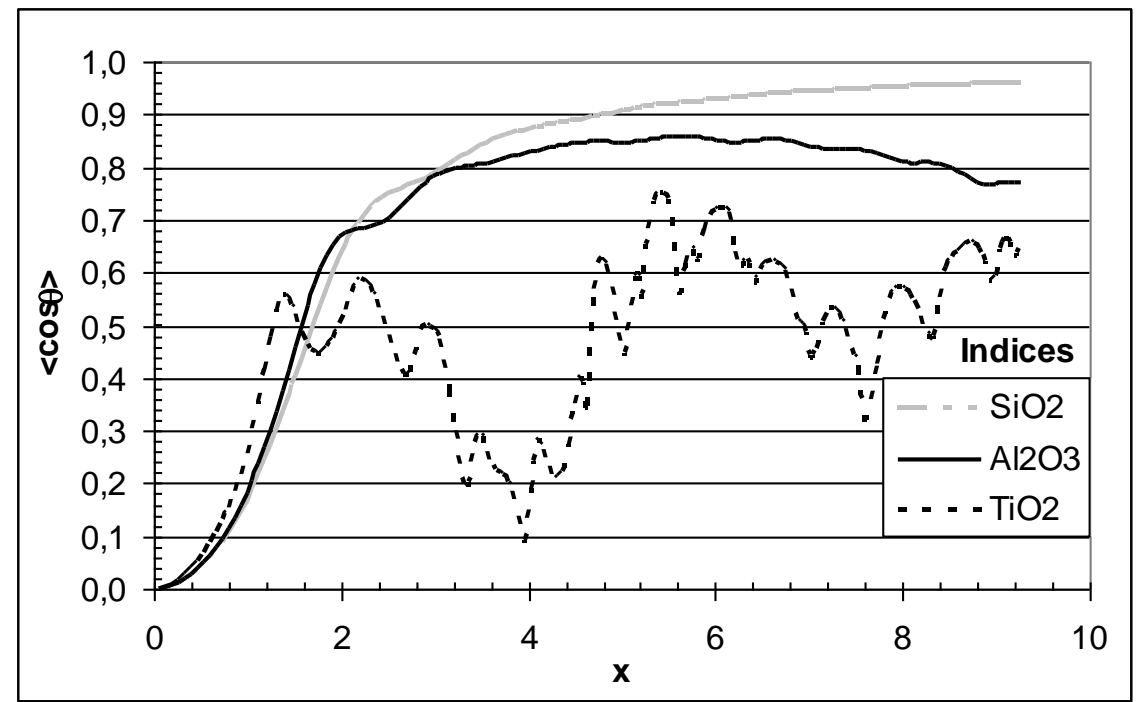

Fig.19: Asymmetry parameter for three materials as function of the size parameter. 
The asymmetry parameter is close to zero for very small size parameters whatever the refractive index. Then, it increases to a plateau close to 1 in the case of $\mathrm{SiO}_{2}$ and $\mathrm{Al}_{2} \mathrm{O}_{3}$, i.e. the light is scattered predominantly in the forward direction. A spherical particle of $\mathrm{TiO}_{2}$ has a mean scattering angle which varies less monotonously according to the size parameter. Indeed, for a size parameter equal to 4 , the asymmetry parameter is close to zero, the scattering can be then described as dipole-like, while for a size parameter of about 6 the scattering seems to happen in a privileged direction.

Fig. 20 represents $C_{p r}$ as a function of the size parameter for a sphere and the three different materials.

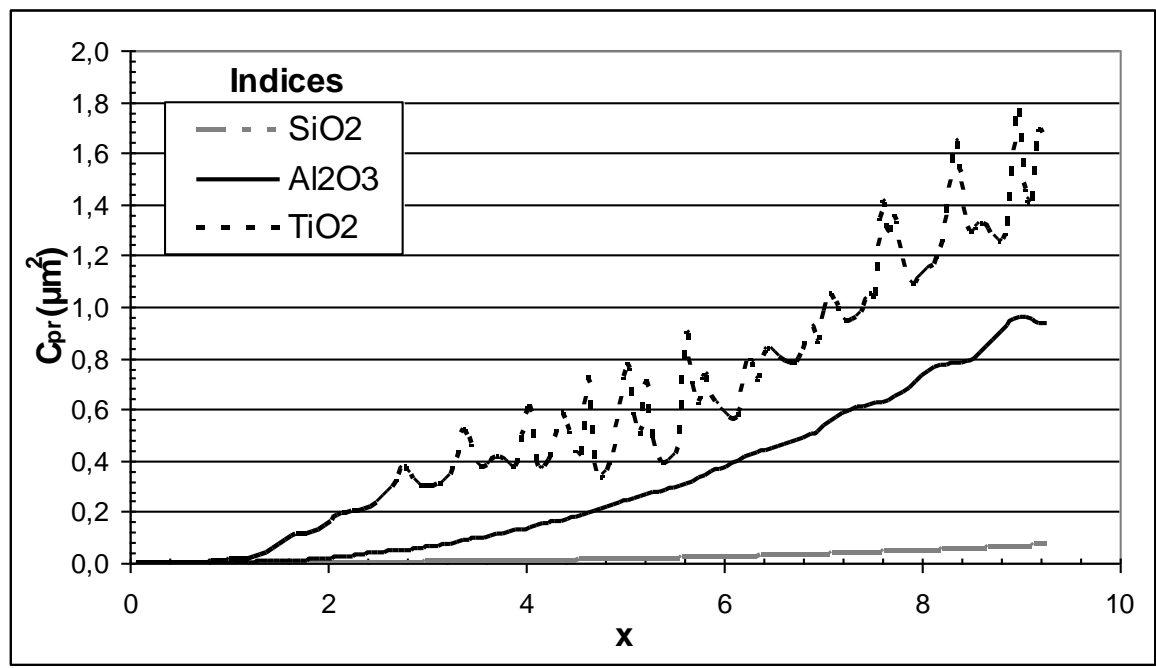

Fig.20: Radiation pressure cross section for three materials as function of the size parameter.

In the size parameter range $[0 ; 10]$, the radiation pressure cross section increases as the optical refractive index increases.

\subsubsection{Aggregate of spheres}

The variations of $C_{p r}$ for an aggregate have been examined according to: the number of primary particles, their size parameter, the relative optical refractive index and the aggregate shape. Simulations were performed by means of GMM method (Xu, 1998b).

The simulation results are presented as previously: effect of the number of primary particles within the aggregate and effect of the aggregate shape on the function $P_{X u}(x) . P_{X u}$ is the ratio 
between $C_{p r}$ value of an aggregate $\left(C_{p r, X u, N}\right)$ and the one of its $N$ primary particles considered as independent $N C_{p r, M i e, 1}$ :

$$
P_{X u}=\frac{C_{p r, X u, N}}{N C_{p r, M i e, 1}}
$$

\subsubsection{Effect of the number of primary particles on radiation pressure cross section}

Fig. 21 represents $P_{X u}$ as a function of the size parameter for a chain-aggregate of $\mathrm{SiO}_{2}$ primary particles. The variation of $C_{p r}$ with primary particle size parameter is similar to the one corresponding to the scattering cross section. Two size parameter ranges can be defined. In the $x$-range $[0 ; 2]$, constructive interferences and multiple scattering (or interaction between primary particles) are important. In the $x$-range [2;10], the radiation pressure cross section is close to the $C_{p r}$ of a set of spheres without interaction. However, multiple scattering yet occurs at some extend.
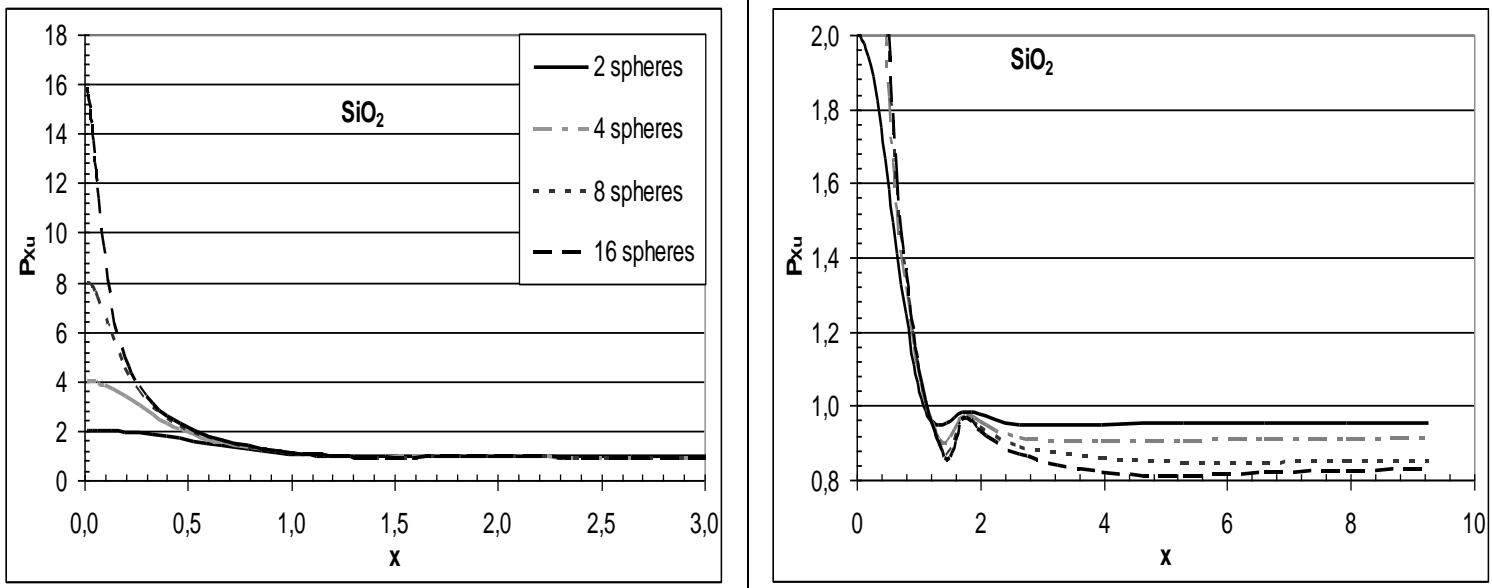

Fig.21: $P_{X u}$ as a function of the size parameter for the linear configuration with $N$ primary particles $(2,4,8,16)$ and for $\mathrm{SiO}_{2}$ : (a) for the range $x \in[0,3]$ and (b) $x \in[0,10]$. 
However, the radiation pressure cross section of an aggregate made up of primary particles whose size parameter is higher than 1.5 seems to be proportional (by a factor $p(N, x) N$ ) to the primary particle radiation pressure cross section. $p(N, x) N$ is a weak function of $x$ for $\mathrm{SiO}_{2}$ (more dependent on $x$ for $\mathrm{TiO}_{2}$ ). As expected, $P_{X u}(x)$ is similar to $K_{X u}(x)$ in the $x$ range $[0 ; 1]$ because the asymmetry factor of the primary particle is smaller than 0.25 . At the contrary, $P_{X u}(x)$ must not be related to $K_{X u}(x)$ in the $x$-range $[1 ; 10]$.

\subsubsection{Effect of the aggregate morphology on radiation pressure cross section}

Figure 22 represents $P_{X u}$ for different configurations of aggregates consisting of 4 primary particles arranged according to Fig 9. Similar variations are obtained. The deviation between the two extreme configurations is about $10.7 \%$, which is a smaller value than that obtained with $C_{s c a}$. But $C_{p r}$ is a little more sensitive to the configuration which are close each other, since the average deviation between the $\mathrm{p} 1$ and $\mathrm{p} 2$ configurations is about $1.3 \%$ compared with $0.8 \%$ for $C_{s c a}$.
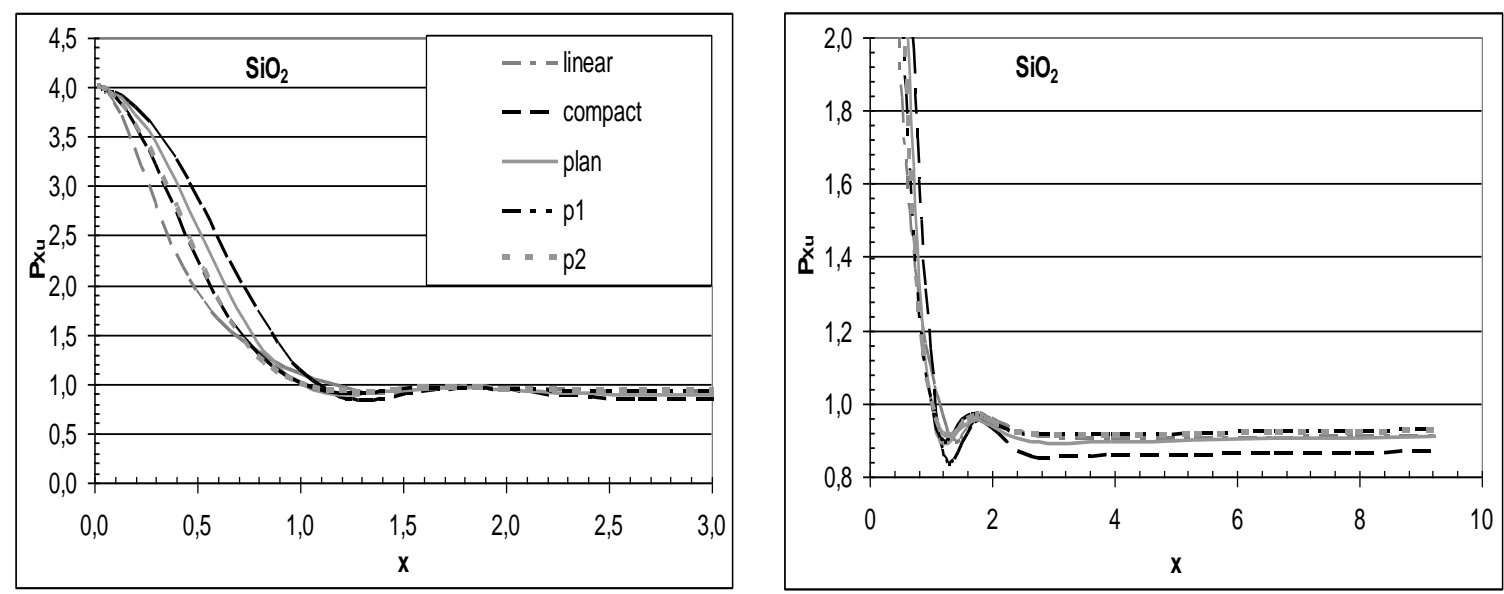

Fig.22: $P_{X u}$ as a function of the size parameter for different configurations of 4-aggregates (linear, compact, plane, p1, p2) for $\mathrm{SiO}_{2}$ 


\subsection{Approximate methods for aggregates}

\subsubsection{ERI method}

In the same way that we showed the effectiveness of the ERI approximate method, for calculation of $\mathrm{C}_{\mathrm{sca}}$, we evaluated (Jacquier and Gruy, 2007b) its performances for the calculation of $C_{p r}$. The ratio of the radiation pressure cross sections obtained on the one hand with the exact (GMM) method and on the other hand with the ERI method is denoted $L_{m}$ :

$L_{m}=\frac{C_{p r, E R I, N}}{C_{p r, X u, N}}$

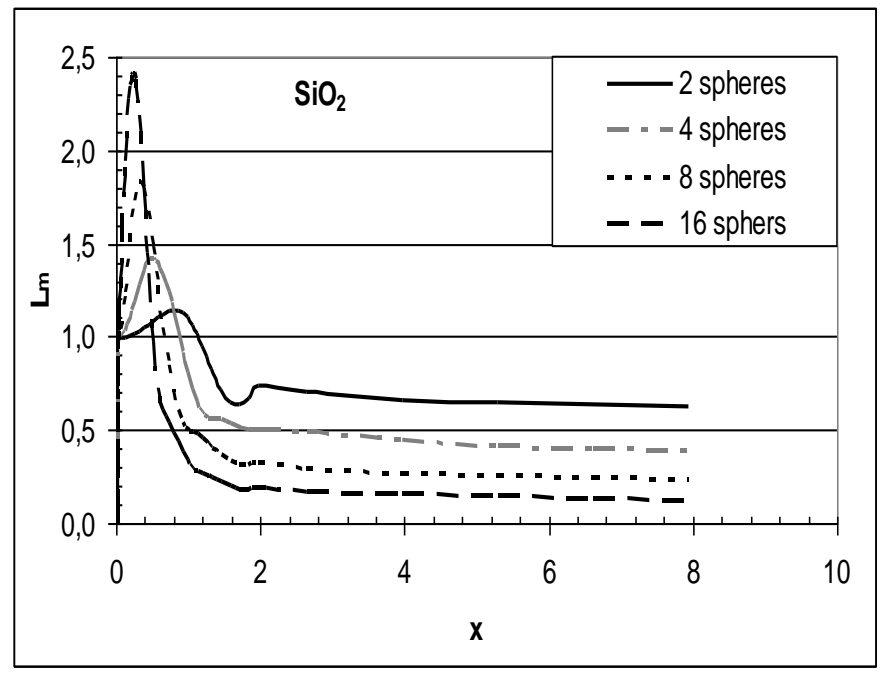

Fig. 23: $L_{m}$ function with $x \in[0 ; 10]$ for linear aggregate $\left(\mathrm{SiO}_{2}\right)$

The deviation of $L_{m}$ from 1 (Fig. 23) leads to the search of a corrective factor in order to reduce it. Jacquier and Gruy (2007b) proposed a corrective factor as a multi-parameter function $G\left(x, N, d_{1} / a\right)$ for the radiation pressure cross section $C_{p r, E R I, N}$. Thus, the corrected radiation pressure cross section obeys the expression:

$C_{p r, E R I, N}^{c o r r}=C_{p r, E R I, N} / G\left(x, N, d_{1} / a\right)$

Later on, this method is called ERI/G.

\subsubsection{Other methods}

We noticed in paragraph 5.2.2 that $P_{X u}$ of any configuration of soft particles does not vary with $x$ for $x$ higher than 1.5. The value of $P_{X u(x>1.5)}$ depends on the aggregate morphology that can be characterized through $N$ and $d_{l} / a$. However, $P_{X u(x>1.5)}$ may be a weak function of $x$ for 
hard material (Fig. 24). We can observe that variations of $P_{X u(x>1.5)}$ are similar to the ones for a two-sphere aggregate.

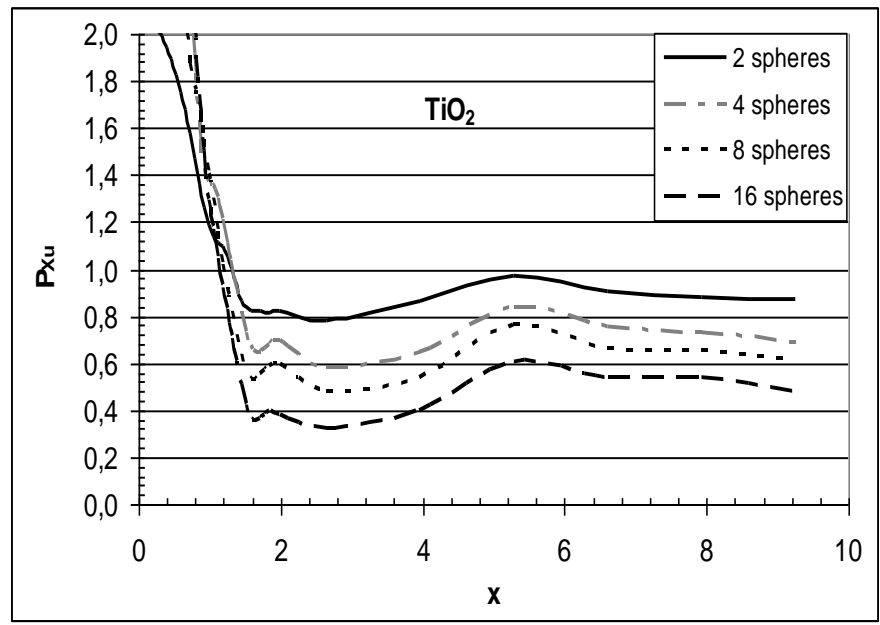

Fig. 24: $P_{X u}$ as a function of the size parameter for the compact configuration with $N$ primary particles $(2,4,8,16)$ and for $\mathrm{TiO}_{2}: x \in[0,10]$.

These comments suggest the two approximate methods:

Method Pp1: $C_{p r X u, N}$ proportional to $C_{p r}$ of a primary particle: $C_{p r M i e, 1}$

Method Pp2: $C_{p r X u, N}$ proportional to $C_{p r}$ of a doublet: $C_{P r X u, 2}$

The proportionality factors, denoted respectively $p_{1}$ and $p_{2}$, can be expressed as a function of a single parameter: $\beta=\frac{N}{\sqrt{d_{1} / a}}$. Corresponding expressions can be found in (Jacquier and Gruy, 2007b). Table 3 presents the performances of ERI/G, Pp1 and Pp2. It appears that the ERI/G method is not as efficient as $\mathrm{Pp} 1$ and $\mathrm{Pp} 2$ but ERI/G presents the biggest advantage to be used over all the size range.

\begin{tabular}{|c|c|c|c|c|c|c|c|c|c|c|c|c|}
\hline \multirow{4}{*}{\begin{tabular}{|l|} 
material \\
method
\end{tabular}} & \multicolumn{12}{|c|}{$1,5<x<10$} \\
\hline & \multicolumn{6}{|c|}{ Linear configuration } & \multicolumn{6}{|c|}{ Compact configuration } \\
\hline & \multicolumn{2}{|l|}{$\mathrm{SiO}_{2}$} & \multicolumn{2}{|c|}{$\mathrm{Al}_{2} \mathrm{O}_{3}$} & \multicolumn{2}{|l|}{$\mathrm{TiO}_{2}$} & \multicolumn{2}{|c|}{$\mathrm{SiO}_{2}$} & \multicolumn{2}{|c|}{$\mathrm{Al}_{2} \mathrm{O}_{3}$} & \multicolumn{2}{|l|}{$\mathrm{TiO}_{2}$} \\
\hline & $\mathrm{m}$ & $\sigma$ & $\mathrm{m}$ & $\sigma$ & $\mathrm{m}$ & $\sigma$ & $\mathrm{m}$ & $\sigma$ & $\mathrm{m}$ & $\sigma$ & $\mathrm{m}$ & $\sigma$ \\
\hline $\mathrm{Pp}_{1}$ & 1,01 & 0,05 & 1,05 & 0,11 & 1,06 & 0,17 & 1,01 & 0,04 & 1,00 & 0,11 & 1,08 & 0,17 \\
\hline $\mathrm{Pp}_{2}$ & 0,98 & 0,04 & 0,96 & 0,08 & 0,94 & 0,12 & 0,97 & 0,04 & 0,93 & 0,10 & 0,93 & 0,11 \\
\hline ERI/G & 0,93 & 0,17 & 0,90 & 0,15 & 1,07 & 0,23 & 0,92 & 0,13 & 0,90 & 0,11 & 1,06 & 0,30 \\
\hline
\end{tabular}


Table 3: Approximate method performance for the calculation of $C_{p r}$ for different material with $x \in[1.5 ; 10] . m$ and $\sigma$ are respectively the mean value and the standard deviation for the corresponding dataset.

\subsection{Conclusion}

We presented different ways to calculate approximately the radiation pressure cross section of aggregates. The corresponding expressions can be used to study dense suspensions. For instance, Tontrup et al. (2000) performed an experimental work about aggregation of $\mathrm{TiO}_{2}$ micro-particles in water by using a backscattering sensor. They deduced the change of the transport mean free path with time. SEM observations showed that the aggregates contain few primary particles. Approximations could be used to determine some characteristics of the aggregates.

\section{Scattering properties versus geometrical parameters of aggregates}

The main question that appears when studying the formation of particles or aggregates is: which is the relevant morphological parameter related to the measurement? The answer mainly depends on the particle size and is partially included in theories and modelling leading to scattering cross section calculations.

So, when we consider the Mie theory for homogeneous spheres, the solving method and the results are only depending on the relative refractive index and the boundary conditions for the Helmholtz equation. From a geometrical point of view, the mathematical function describing the particle surface is the relevant parameter. The case of non-spherical convex bodies is similar. As the physics is always based on Maxwell and Helmholtz equations, the corresponding solution for a natural incident light only depends on the body surface that is characterized by the equation $f(x, y, z)=0$.

If we are interested in the orientation average of the scattering cross section, a function describing the body and being invariant to rotation will be preferred. So, the pair distance distribution density (PDDD) could be an interesting approach to describe the shape. It is a well known function in physics and can be defined for liquids as:

$d N=g(r) 4 \pi r^{2} d r$ 
$d N$ represents the number of molecules distant from a given (tagged) molecule with the distance in the range $[r, r+d r]$. In the case of liquid, the distribution is nearly isotropic. This function clearly appears in RGD approximation for convex bodies (distribution density is connected to $|f|^{2}$ ) and BPK approximation for multi-sphere aggregate (in Eq.14a,b).

In the first case (RGD), we consider any pair of volume elements in the scattering body. The pair distance distribution density is a continuous function of the distance between volume elements. In our context, we chose the notation $D_{P}(r)$. Then, the orientation averaged scattering cross section can be written as (Gruy, 2009):

$C_{R G D, 1}=\frac{2}{3 \pi} k^{4} V^{2}(m-1)^{2} \int_{R_{\min }}^{R_{\max }} F(k r) D_{P}(r) d r$

with

$F(x)=3\left[\cos (2 x)\left(-1+5 x^{-2}-3 x^{-4}\right)+\sin (2 x)\left(2 x^{-1}-6 x^{-3}\right)+1+x^{-2}+3 x^{-4}\right] /\left(4 x^{2}\right)$

The distribution density function is normalized:

$\int_{R_{\min }}^{R_{\max }} D_{P}(r) d r=1$

Figure 25 presents the function $D_{P}(r)$ for a sphere and various spheroids. The pair distribution function for a sphere with radius $a$ obeys the expression:

$a D_{p}(r)=D_{p, u}(u)=\frac{3}{16} u^{2}\left(u^{3}-12 u+16\right)$

with

$u=r / a \quad$ and $\quad 0<u<2$ 


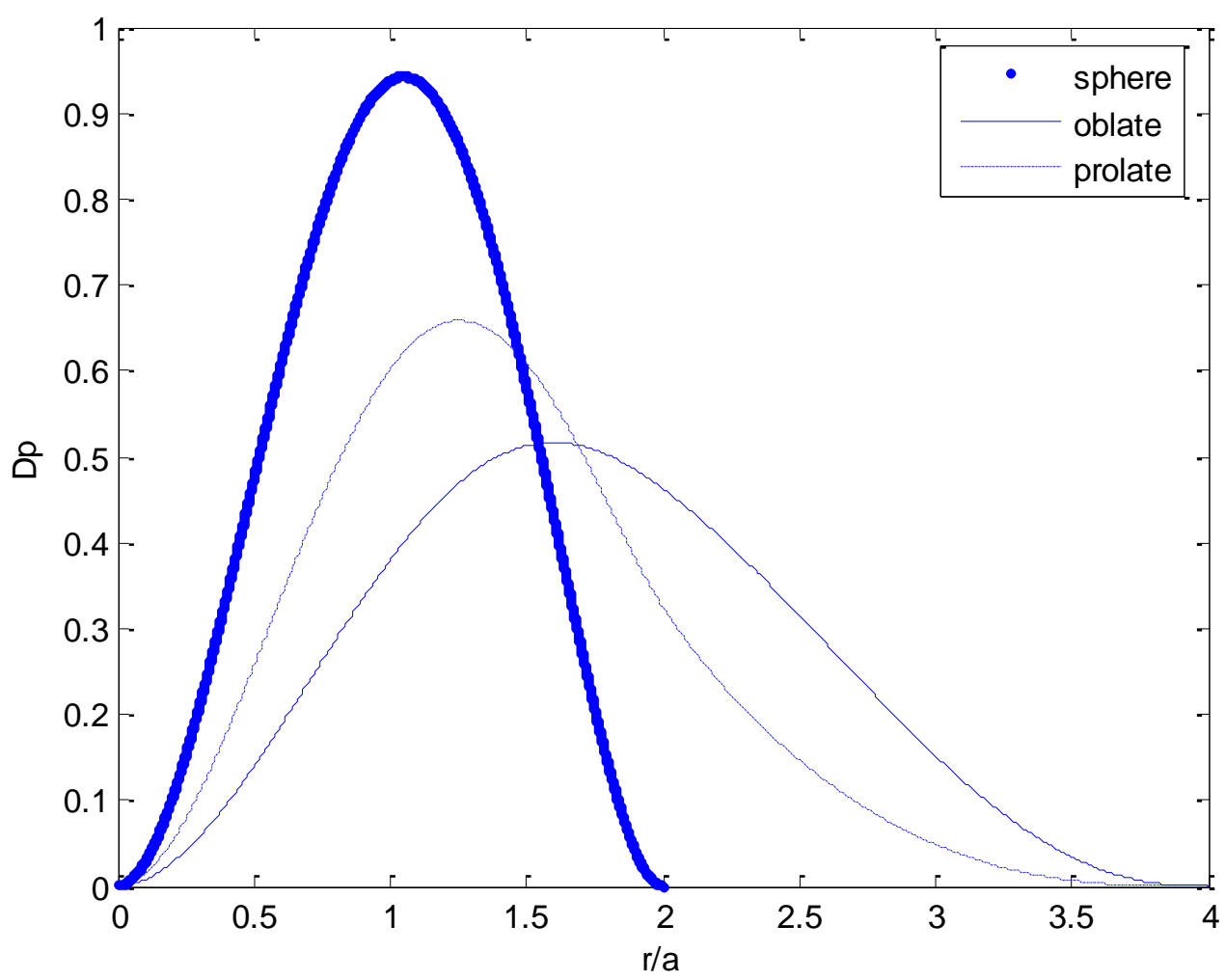

Fig.25: Pair distance distribution density for sphere, oblate spheroid (axis ratio equal to 2) and prolate spheroid (axis ratio equal to 2). $a$ is the smallest semi-axis length.

In the second case (BPK), equations contain the inter-particle distance $d_{i j}$. This function is not continuous; as far as a cluster of point scatterers is concerned:

$D_{P}(r)=\frac{1}{N(N-1)} \sum_{i, j} \delta\left(r-d_{i j}\right)$

$\delta$ is the Dirac function.

A particular case is the fractal-like aggregate, the PDDD of which obeys the equation (continuous form):

$$
D_{P}(r) \propto r^{D_{F}-3}
$$

According to paragraph 4.3.2, the BPK approximation gives good results when the size parameter of the primary particle is smaller than 2. Thus, the PDDD is the relevant morphological parameter.

It has been shown by Gruy (2009) that this function associated with BP approximation (Berry and Percival, 1986) for aggregates of Rayleigh scatterers allows for an estimation of the 
scattering cross section of convex bodies. This method is proved efficient for spheres and spheroids as the scattering efficiency is smaller than 1 and as the material is optically either soft or hard.

Non corrected ERI method is only based on the average projected area $\left\langle S_{p}\right\rangle_{O}$ of the body. However, it is not suitable for elongated aggregates with small size parameter. $\left\langle S_{p}\right\rangle_{O}$ can be expressed as a function of the number of primary particles and of the mean inter-particle distance (Jacquier and Gruy, 2008a,b):

$<S_{p}>_{O}=\pi R_{p}^{2}$

with

$R_{p}=a\left(d_{1} / 2 a\right)^{1 / 5} N^{1 / 3} d_{E}^{1 / 8} \quad$ and $N<100$,

$d_{E}$ is the space dimension.

$d_{1}$ is the relevant morphological parameter. It is directly related to the first moment of the distribution $D_{P}(r)$ :

$M_{1}=\int_{0}^{\infty} D_{P}(r) r d r=\int_{0}^{\infty} \frac{1}{N(N-1)} \sum_{i, j} \delta\left(r-d_{i j}\right) r d r=\frac{1}{N(N-1)} \sum_{i, j} d_{i j}=d_{1}$

It would be possible to choose other moments of the distribution for describing geometrical and optical properties of aggregates. For instance, the second order moment is directly related to the gyration radius, that is a well known parameter used to define a fractal-like aggregate. However, there was no noticeable change and thus no improvement was found when choosing another mean distance definition for the aggregate. Thus, we chose the lowest order distribution moment. Corrected ERI method also uses $d_{l}$ distance parameter.

For large size parameter $(x>2), \mathrm{AD}$ approximation becomes efficient. In this case, the relevant line is the chord. Expression of the average scattering cross section can be re-written by introducing the chord length distribution $D_{l}(l)$ (Jacquier and Gruy, 2008a,b):

$$
\left\langle C_{A D, N}\right\rangle=\left\langle 2 \iint_{\left[S_{p}\right]}(1-\cos k l(m-1)) d y d z\right\rangle \square 2<S_{p}>\int_{0}^{l_{\max }}(1-\cos k l(m-1)) D_{l}(l) d l
$$

The chord length distribution (CLD) is defined as follows: $D_{l}(l) d l$ represents the number fraction of the chord length in the range $[l, l+d l]$. Thus, $D_{l}(l)$ obeys the normalization equation: 
$\int_{0}^{l_{\max }} D_{l}(l) d l=1$

$l_{\max }$ is the maximum chord length of an aggregate.

The figure 26 presents the $D_{l}(l)$ function for an aggregate consisting of 16 primary particles. One observes three very distinct peaks or modes, each one characterized by a chord length range:

- $\quad[0 ; 2 \mathrm{a}]$ corresponds to primary particles (distribution density $D_{l, 1}(l)$ )

- $\quad[2 \mathrm{a} ; 4 \mathrm{a}]$ corresponds to pair of particles in contact (distribution density $D_{l, 2}(l)$ )

- $\quad\left[4 \mathrm{a} ; 1_{\max }\right]$ corresponds to the aggregate superstructure (distribution density $D_{l, G}(l)$ )

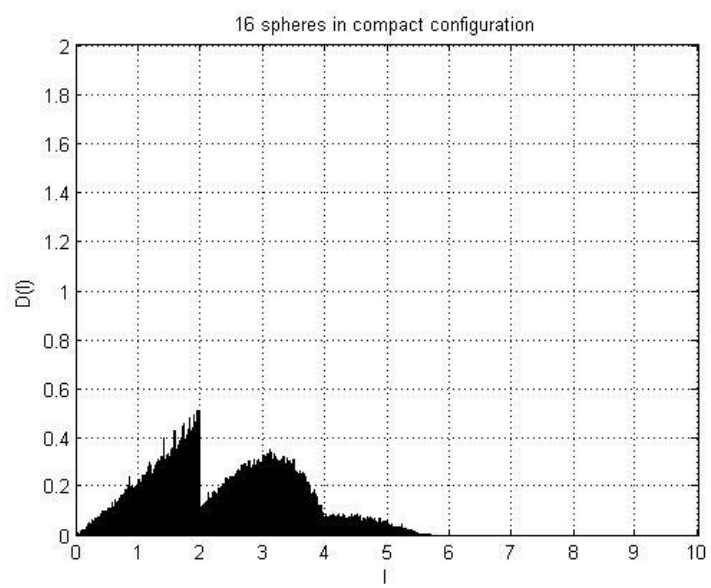

Fig. 26: $D_{l}(l)$ for compact aggregate with 16 primary particles. $l$ is normalized by the radius of the primary particle.

$D_{l}(l)$ contains the contributions of each distribution density. These are weighted by the coefficients $\xi_{1}, \xi_{2}, \xi_{G}$ :

$$
D_{l}(l)=\xi_{1} D_{l, 1}(l)+\xi_{2} \frac{D_{l, 2}(l \in[2 a ; 4 a])}{\int_{2 a}^{4 a} D_{l, 2}(l) d l}+\xi_{G} \frac{D_{l, G}\left(l \in\left[4 a ; l_{\max }\right]\right)}{\int_{4 a}^{l_{\max }} D_{l, G}(l) d l}
$$

with

$\xi_{1}+\xi_{2}+\xi_{G}=1$

The distribution densities $D_{l, 1}(l)$ and $D_{l, 2}(l)$ are given by analytical expressions (Jacquier and Gruy, 2008). $D_{l, G}(l)$ is an empirical function, the same for all the aggregates. Only weighting coefficients depend on the aggregate morphology. Results shown in Fig. 26 for a particular 
aggregate are representative for aggregates with primary particle number up to one hundred. Coefficients $\xi_{1}, \xi_{2}, \xi_{G}$ depend on $N, d_{1}, d_{E}$ (Jacquier and Gruy, 2008).

The calculation of the scattering cross section using Eqs.32-34a,b is much faster than that based on Eq.19.

Certain presented approximations are characterized by a decoupling or separation between optics and geometry. This separation allows for a faster calculation of the optical properties. At our knowledge, the relationship between chord length distribution (as defined by figure 15) and pair distance distribution is not trivial, particularly for aggregates, and requires complementary works in the field of integral geometry. Moreover, the transition between the different geometrical characteristics, i.e PDD and CLD, is not yet quantitatively understood as the primary particle size increases.

\section{Conclusion}

The analysis of turbidimetric data during precipitation process is a challenge for researchers working in the field of light scattering by particles. The variety of sizes, shapes and optical contrast requires several approaches for calculation of their optical properties. Performance criteria are the calculation speed and the accuracy fitted with the measurement one. Accurate calculations performed with numerical sophisticated methods will be always needed and used for the purpose of validation. Difficulties remain for certain particles with a complex morphology. For instance, one observes precipitated zinc sulphide particles in the size range $[0.5 \mu \mathrm{m} ; 5 \mu \mathrm{m}]$ exhibiting sand rose (i.e., gypsum flower) morphology. The typical multi-scale morphology of many precipitated particles firstly needs tools coming from the integral geometry in order to be described with a minimum number of parameters. Knowing this parameter set, optical properties will be calculated with exact theories. The parameter number coming from geometrical analysis can be reduced when the optical properties of the particles are appropriately considered for the formulation of approximate theories to the calculation of scattering properties. An example for such an approach has been presented, but further advances are needed.

\section{Acknowledgment}

The authors thank Frank Babick from Technical University of Dresden (Germany) for the helpful discussions and the final revision of this text. 


\section{References}

Akkermans, E., P.E.Wolf, R. Maynard, G. Maret, 1988: Theoretical study of the coherent backscattering of light by disordered media, J. Phys. Fr., 49, 77-98.

Asano, S., 1979: Light scattering properties of spheroidal particles, App.l Optics, 18(5), $712-$ 723.

Asano, S. and M. Sato, 1980: Light scattering by randomly oriented spheroidal particles." Appl. Optics, 19(6), 962-974.

Asano, S. and G. Yamamoto, 1975: Light scattering by spheroidal particle, Appl. Optics, 14(1), 29-49.

Auger, J.-C., R. G. Barrera, B. Stout, 2003: Scattering efficiency of clusters composed by aggregated spheres, J. Quant. Spectr. Rad. Transfer, 79-80, 521-531.

Auger, J.-C., B. Stout, V. Martinez, 2005: Scattering efficiency of aggregated clusters of spheres: dependence on configuration and composition, J. Opt. Soc. Am., 22(12), 2700-2708.

Berry, M. V. and I. C. Percival, 1986: Optics of fractal clusters such as smoke, Opt. Acta, 33(5), 577-591.

Bohren, C. F. and D. R. Huffman, 1998: Absorption and scattering of light by small particles, Wiley-VCH, Berlin.

Cameirao, A., R. David, F. Espitalier, F. Gruy, 2008: Effect of precipitation conditions on the morphology of strontium molybdate agglomerates, J. Cryst. Growth, 310, 41524162.

Chylek, P. and J. D. Klett, 1991: Absoption and scattering of electromagetic radiation by prismatic columns: anomalous diffraction approximation, J. Opt. Soc. Am., 8, 274281.

Coccioli, R., T. Itoh, G. Pelosi, P. P. Silvester, 1996: Finite elements methods in microwaves: a selected bibliography, Antennas Propag. Mag., 38, 34-48.

Coudun, C., E. Amblard, J-C Guihaumé and J-F Hochepied, 2007: Nanostructured particles by controlled precipitation techniques; example of nickel and cobalt hydroxides, Catal. Today, 124, 49-54.

de Wolf, D.A.,1991: Backscatter enhancement: random continuum and particles, J. Opt. Soc. Am. A, 8, 465-471.

Draine, B. T. and P. J. Flatau, 1994: Discrete-dipole approximation for scattering calculations, J. Opt. Soc. Am., 11(4), 1491-1499.

Farafonov, V. G., V. B. Il'in, T. Henning, 1999: A new solution of the light scattering problem for axisymmetric particles, J. Quant. Spectr. Rad. Transfer, 63, 205-215.

Elimelech, M., J. Gregory, X. Jia, R. Williams, 1995: Particle deposition and aggregation, measurement, modelling and simulation, Butterworth-Heinemann Ltd, Oxford.

Fuller, K. A. and G. W. Kattawar, 1988a: Consummate solution to the problem of classical electromagnetic scattering by an ensemble of spheres.I: Linear chains, Opt. lett., 13(2), 90-92.

Fuller, K. A. and G. W. Kattawar, 1988b: Cosummate solution to the problem of classical electromagnetic scattering by an ensemble of spheres II: Clusters of arbitrary configuration, Opt. lett., 13(12), 1063-1065.

Gruy, F., 2001: Formation of small silica aggregates by turbulent aggregation, J. Colloid Interf. Sci, 237, 28-39. 
Gruy, F. and S. Jacquier, 2008: The chord length distribution of a two-sphere aggregate, Comp. Mater. Sc., 44, 218-223.

Gruy, F., 2009: Light scattering cross section as a function of pair distribution density, $J$. Quant. Spectr. Rad. Transfer, 110, 240-246.

Harrington., R. F., 1968: Field computation by moment methods, New York, Macmillan.

Helfenstein, P., J. Ververka, J. Hillier, 1997: The lunar opposition effect, a test of alternative models, Icarus, 128, 2-14.

Hovenier, J. W., K. Lumme, M. I. Mishchenko, N. V. Voshchinnikov, D. W. Mackowski, J. Rahola, 1996: Computations of scattering matrices of four types of non-sphericles using diverse methods, J. Quant. Spectr. Rad. Transfer, 55(6), 695-705.

Iati, M.A., R. Saija, A. Giusto, P. Denti, F. Borghese, C. Cecchi-Pestellini, 2004: Optical properties of interstellar gain aggregates, J. Quant. Spectr. Rad. Transfer, 89, 43-45.

Ishimaru, A., 1978: Wave Propagation and scattering in random media (vol. 1 and 2), Academic Press, New-York.

Ishimaru, A., Y. Kuga, R.L.T. Cheung, K. Shimizu, 1983: Scattering and diffusion of a beam wave in randomly distributed scatterers, J. Opt. Soc. Am., 73(2), 131-136

Jacquier, S., 2006: Approximate methods for the optical properties of spherical non-absorbent aggregated particles. PhD thesis, Ecole Nationale Supérieure des Mines de SaintEtienne, Saint-Etienne.

Jacquier, S. and F. Gruy, 2007a: Approximation of the light scattering cross-section for aggregated spherical non-absorbent particles, J. Quant. Spectr. Rad. Transfer, 106, 133-144.

Jacquier, S. and F. Gruy, 2007b: Approximation for scattering properties of aggregated spherical particles, PARTEC 2007, Nürnberg.

Jacquier, S. and F. Gruy, 2008a: Anomalous Diffraction Approximation for light scattering cross section: case of ordered clusters of non-absorbent spheres, J. Quant. Spectr. Rad. Transfer, 109, 789-810.

Jacquier, S. and F. Gruy, 2008b: Anomalous Diffraction Approximation for light scattering cross section: case of random clusters of non-absorbent spheres, J. Quant. Spectr. Rad. Transfer, 109, 2794-2803.

Kahnert, F. M., 2003: Numerical methods in electromagnetic scattering theory, J. Quant. Spectr. Rad. Transfer, 79(80), 775-824.

Khlebtsov, N. G., 1996: Spectroturbidimetry of fractal clusters: test of density correlation function cut-off, Appl. Optics, 35(21), 4261-4270.

Kimura, H. and I. Mann, 1998: Radiation pressure cross section for fluffy aggregates $J$. Quant. Spectr. Rad. Transfer, 60(3), 425-438.

Kimura, H., H. Okamoto, T. Mukai, 2002: Radiation pressure and the Pointing-Robertson effect for fluffy dust particles, Icarus, 157, 349-361.

Kokhanovsky A.A., 2001: Light scattering media optics: problems and solutions (Second Edition), Praxis Publishing Ltd., Chichester.

Kolokolova, L. and B. A. S. Gustafson, 2001: Scattering by inhomogeneous particles: microwave analog experiments and comparison to effective medium theories, $J$. Quant. Spectr. Rad. Transfer, 70, 611-625.

Kostoglou M., A.G. Konstandopoulos, S.K. Friedlander, 2006: Bivariate population dynamics simulation of fractal aerosol aggregate coagulation and restructuring, Aerosol Sci., 37(9), 1102-1115.

Kruyt, H.R., 1952: Colloid science, Elsevier Publishing Company, Amsterdam.

Kuga, Y. and A.Ishimaru, 1984: Retroreflectance from a dense distribution of spherical particles, J. Opt. Soc. Am.,1(8), 831-835. 
Liu, C.-L., 1998: Validity of anomalous diffraction approximation in m-X domain, Atmos. Res., 49, 81-86.

Liu, Y., W. P. Arnott, J. Hallett, 1998: Anomalous diffraction theory for arbitrarily oriented finite circular cylinders and comparison with exact T-matrix results, Appl. Optics, 37(21), 5019-5030.

Lopatin, V.N. and F.Ya. Sid'Ko, 1988: Introduction to optics of cell suspensions, Moscow, Nauka.

Mekki-Berrada K., F. Gruy, M. Cournil, 2005 : Synthèse d'agglomérats multi-échelles par précipitation homogène (Récents Progrès en Génie des Procédés), Edition Lavoisier, Paris.

Mishchenko, M. I., D. W. Mackowski, L. D. Travis, 1995: Scattering of light by bispheres with touching and separated components, Appl. Optics, 34(21), 4589-4599.

Mishchenko, M. I., L. D. Travis, and A. A. Lacis, 2002: Scattering, Absorption, and Emission of Light by Small Particles, Cambridge University Press, Cambridge.

Mishchenko, M. I., G. Videen, V. A. Babenko, N. G. Khlebtsov, T. Wriedt, 2004: T-matrix theory of electromagnetic scattering by particles and its applications: a comprehensive reference database, J. Quant. Spectr. Rad. Transfer, 88, 357-406.

Mishchenko, M. I., G. Videen, V.A. Babenko, N.G. Khlebtsov, T. Wriedt , 2007 : Comprehensive T-matrix reference database: A 2004-06 update, J. Quant. Spectr. Rad. Transfer, 106, 304-324.

Mishchenko, M. I., G. Videen, N.G. Khlebtsov, T. Wriedt, N.T. Zakharova, 2008: Comprehensive T-matrix reference database: A 2006-07 update, J. Quant. Spectr. Rad. Transfer, 109, 1447-1460.

Nichols, M.G., E.L. Hull, T.H. Forster, 1997: Design and testing of a white-light, steady-state diffuse reflectance spectrometer for determination of optical properties of highly scattering systems, Appl. Optics, 36, 93-104.

Quirantes, A., F. Arroyo, et al., 2001: Multiple light scattering by spherical particle systems and its dependence on concentration; a T-matrix study, J. Colloid Interf. Sci., 240, 78-82.

Randolph, A. D., and M.A. Larson, 1988: Theory of Particulate Processes, Academic Press, NewYork.

Rouleau, F., 1996: Electromagnetic scattering by compact clusters of spheres, Astron. Astrophys., 310, 686-698.

Streekstra G.J., A. G. Hoekstra, et al., 1994: Anomalous diffraction by arbitrarily oriented ellipsoids: applications in ektacytometry, Appl. Optics, 33, 7288-7296.

Sugimoto T., 2000: Fine particles: synthesis, characterization, and mechanisms of growth, (Surfactant science series Vol 92), Marcel Dekker, New York.

Sun, W. and Q. Fu, 1999: Anomalous diffraction theory for arbitrarily oriented hexagonal crystals, J. Quant. Spectr. Rad. Transfer, 63, 727-737.

Sun, W. and Q. Fu, 2001: Anomalous diffraction theory for randomly oriented nonspherical particles: a comparison between original and simplified solutions, J. Quant. Spectr. Rad. Transfer, 70, 737-747.

Tandon P., D.E. Rosner, 1999: Monte Carlo Simulation of Particle Aggregation and Simultaneous Restructuring, J. Colloid and Interf. Sci., 213, 273-286.

Tontrup C., F. Gruy and M. Cournil, 2000: Turbulent aggregation of titania in water, J. Colloid and Interf. Sci., 229, 511-525.

Tsang L., A. Ishimaru, 1984: Backscattering enhancement of random discrete scatterers, J. Opt. Soc. Am., 1, 836-839. 
Tsang L., A. Ishimaru, 1985: Theory of backscattering enhancement of random discrete isotropic scatterers based on the summation of all ladder and cyclical terms, J. Opt. Soc. Am. A, 2, 1331-1338.

Van de Hulst H. C., 1981: Light scattering by small particles, Dover publications Inc., New York.

Videen, G. and P. Chylek, 1998: Anomalous diffraction approximation limits, Atmos. Res., 49, 77-80.

Voshchinnikov, N. V. and V. G. Farafonov, 1992: Optical properties of spheroidal particles, Astrophys. Space Sci., 204,19-86.

Wolf, P.E., G. Maret, E. Akkermans, R. Maynard, 1988: Optical coherent backscattering by random media: an experimental study, J. Phys. Fr., 49, 63-75.

Wriedt, T., 1998: A review of elastic light scattering theories, Part. Part. Syst. Charact., 15, 67-74.

Xu, Y.-L., 1995: Electromagnetic scattering by an aggregate of spheres, Appl. Optics, 34(21), 4573-4588.

$\mathrm{Xu}$, Y.-L., 1996: Calculation of the addition coefficients in electromagnetic multispherescattering theory, J. Comput. Phys., 127, 285-298.

Xu, Y.-L., 1997a: Electromagnetic scattering by an aggregate of spheres: far field, Appl. Optics, 36(36), 9496-9508.

Xu, Y.-L., 1997b: Fast evaluation of gaunt coefficients: recursive approach, J. Comput. Appl. Math., 85, 53-65.

Xu, Y.-L., 1998a: Efficient evaluation of vector translation coefficients in multiparticle lightscattering theories, J. Comput. Phys., 139, 137-165.

$\mathrm{Xu}$, Y-L, 1998b: Electromagnetic scattering by an aggregate of spheres: asymmetry parameter, Phys. Lett. A, 249, 30-36

Xu, Y.-L. and B. A. S. Gustafson, 2001: A generalized multiparticle Mie-solution: further experimental verification, J. Quant. Spectr. Rad. Transfer, 70, 395-419.

Yang, P. and K. Liou, 2000: Finite difference time domain method for light scattering by nonspherical and inhomogeneous particles. In Light Scattering by Nonspherical Particles: Theory, Measurements, and Applications, eds. M. I. Mishchenko, J. W. Hovenier, and L. D. Travis., Academic Press, San Diego.

Yang, P., Z. Zhang, B. A. Baum, H. L. Huang, Y. X. Hu, 2004: A new look at anomalous diffraction theory (ADT): Algorithm in cumulative projected-area distribution domain and modified ADT, J. Quant. Spectr. Rad. Transfer, 89, 421-442. 